TRANSACTIONS OF THE

AMERICAN MATHEMATICAL SOCIETY

Volume 249, Number 2, May 1979

\title{
NONCOLLISION SINGULARITIES IN THE FOUR-BODY PROBLEM
}

\author{
BY
}

\author{
ROBERT ORRIN SHELTON
}

\begin{abstract}
It is shown that if there is a singularity in a solution of the four-body problem which is not a collision then the motion of the bodies near the singularity is nearly one-dimensional. This is established by grouping the bodies into natural clusters and showing the angular momentum of each cluster with respect to its center of mass tends to zero near the singularity. This is related to Sperling's proof of von Zeipel's theorem.
\end{abstract}

Introduction. A long-standing open problem in celestial mechanics is to describe the singularities which may occur in solutions to the equations of the $n$-body problem. Several pieces of this problem have been resolved. For example, it is known that in the two cases, $n=2$ or $n=3$, any singularity results from a collision between two or more particles. A collision is said to be any singularity in which all particles tend to limiting positions at the singularity. This idea of a collision singularity includes the fact that two or more of the limiting positions must be equal, for if not, the solution would not have a singularity at the prescribed time.

It is not known in general if all singularities have the property that the positions of the particles tend to limits at the singularity. Classical and current results will be described in the following paragraphs. One of the first tools developed to treat the general question of describing singularities of the $n$-body problem is a lemma of P. Painlevé [1] (Lemma 1 of this paper). Painleve showed that the potential energy tends to infinity at any singularity of the $n$-body problem. One may apply this result to show that all singularities of the 3-body problem are collisions.

A somewhat different theorem was proposed and partially proved by $\mathbf{H}$. von Zeipel [2]. The proof was completed in 1968 by Hans Sperling [3]. This result is that the maximum distance between particles tends to infinity at a noncollision singularity. A very important tool in the study of noncollision singularities which is used in the proof of von Zeipel's result is the idea of grouping the $n$ particles into natural clusters. This idea of clustering is used extensively in this paper, but the method used to form the clusters differs from the "counting argument" used by Sperling. This clustering process and

Presented to the Society, November 23, 1974; received by the editors July 27, 1976.

AMS (MOS) subject classifications (1970). Primary 70F10. 
the behavior of these clusters is studied rigorously in this paper, so that a relatively detailed picture of a noncollision singularity in the 4-body problem is given.

The present work considers solutions of the equations of the four-body problem which may contain binary collisions. Theorem 2 asserts that such a solution which is a noncollision singularity must collapse to a line at the time of the singularity, i.e. the motion becomes essentially one dimensional near a noncollision singularity. This means that all of the bad behavior of the positions of the four particles may be confined to a certain direction in space. This is proved by grouping the masses into natural clusters and proving that the angular momentum of any cluster tends to 0 at a noncollision singularity; this is Theorem 1.

These results suggest that all singularities are due to collisions. A joint paper of R. McGehee and J. Mather [4] strongly indicates that noncollision singularities may in fact occur. They construct a function which solves the equations of the 4-body problem, transformed to regularize binary collisions, and which is a noncollision singularity. The present work, which was done in ignorance of the work of McGehee and Mather, suggests that their construction could only succeed in this linear case.

Classically speaking, this function is not a noncollision singularity. In fact, it contains infinitely many binary collisions. On the other hand, it may be possible to find a solution of the 4-body problem which is close enough to the construction of McGehee and Mather to be a noncollision singularity. It is worth mentioning that McGehee and Mather constructed their solution by taking the particles to lie on a line.

\section{Basic Treatment of Singularities in the $\boldsymbol{n}$-Body Problem}

$\S 1$ includes a general discussion of the $n$-body problem with emphasis on the behavior of singularities. $\S \S 2$ and 3 are the beginning of a careful description of the behavior of a noncollision singularity in the 4-body problem, which includes a detailed explanation of the clustering process (Lemmas 3, 4, 5 and 7). The material in $\$ 1$ is completely standard, and some of the ideas underlying the work in $\$ \S 2$ and 3 are due to Saari [5].

1. In this section the $n$-body problem is stated precisely as a system of equations are given without proof. The section ends with two basic lemmas. Both Lemma 1 and Lemma 2 crudely describe the behavior of the potential near the time of a singularity in the equations of the $n$-body problem. near the time of a singularity in the equations of the $n$-body problem.

Notation. Let $R_{i}$ be the elements of $R^{3}$ and $m_{i}$ be positive numbers for $i=1, \ldots, n$. Let $R_{i j}=R_{i}-R_{j}$ and $r_{i j}=\left|R_{i j}\right|$. We shall use $\Sigma$ for a 
summation over $i, i=1, \ldots, n$, and $\Sigma^{\prime}$ for a summation over $j$ for fixed $i$ and $j=1, \ldots, n$ but $j \neq i$.

EQuATION. The $n$-body problem is described by the equations

$$
R_{i} \cdot=-\sum^{\prime} m_{j} R_{i j} / r_{i j}^{3}, \quad i=1,2, \ldots, n .
$$

Classical DEFINITION OF SINGUlarity. The right members of the equations of the $n$-body problem are analytic functions of $R_{i}, i=1, \ldots, n$, as long as the distances $r_{i j}$ are bounded away from 0 . Cauchy's theorem for ordinary differential equations states that given $R_{i}\left(t_{0}\right)=R_{0 i}, R_{i}\left(t_{0}\right)=V_{0 i}, i=$ $1, \ldots, n, \min _{1<i<j<n} r_{i j}\left(t_{0}\right)=a>0$ and $\max _{1<i<n}\left|V_{0 i}\right|=b$, then there exists a solution $R_{i}(t), R_{i}(t)$ with $R_{i}\left(t_{0}\right)=R_{0 i}, R_{i}\left(t_{0}\right)=V_{0 i}$. Moreover, this solution is unique and analytic for $\left|t-t_{0}\right|<\delta$, and $\delta$ depends on $a, b, m_{i}$. A point $t^{*}$ is a point of singularity of a solution if $R_{i}(t), R_{i}(t)$ analytic for $t$ in some interval $\left(t^{*}-\varepsilon, t^{*}\right)$ and $R_{i}, R_{i}$ may not be continued analytically past $t^{*}$.

Collisions. If $t^{*}$ is a point of singularity of a solution, $R_{i}, R_{i}$ of the $n$-body problem and the limit from the left of $R_{i}(t)$, as $t$ approaches $t^{*}, i=1, \ldots, n$, exists, then the singularity is said to be a collision. There are many examples of collision singularities. General collision or total collapse is a solution with $\lim _{t \rightarrow t^{*}} R_{i}(t)=0$. An example of some initial conditions which produce such a collision is a planar solution with equal masses and

$$
\begin{gathered}
R_{i}\left(t_{0}\right)=\left(r_{0} \cos (2 \pi(i / n)), r_{0} \sin (2 \pi(i / n))\right) \\
R_{i}\left(t_{0}\right)=0, \quad i=1, \ldots, n .
\end{gathered}
$$

BINARY COLLISIONS. A singularity which is due to the coincidence of one or more separate pairs of masses is called a binary collision. In the classical sense, binary collisions are singularities. On the other hand, it is well known that such singularities may be removed by suitable transformations of the space and time variables. Thus a solution which "ends" in a binary collision at time, $t^{*}$, may be extended through $t^{*}$ so that the branches of the solution to the left and right of $t^{*}$ match in every possible sense. For a full treatment of these facts, one should consult the works of Sundman and more recently, Moser and Siegel [6].

For the remainder of this paper, we assume that any solution which would end with a binary collision has been extended. It is important to note that the set of $t$ at which binary collisions occur on a given orbit is discrete. Naturally the functions, $R_{i}, R_{i}$, are analytic except at the times of the collisions and the functions $R_{i}$ are continuous through the collisions.

NonCOLlision SINGULARITIES. A solution of the $n$-body problem is said to have a noncollision singularity at time $t^{*}$ if one or more $R_{i}, i=1, \ldots, n$, has no limit at $t^{*}$. Noncollision singularities cannot occur when $n \leqslant 3$ and it is a 
long-standing open question whether they indeed can occur with more bodies.

STANDARD FORMULAS AND CONSTANTS OF MOTION. Without loss of generality assume that the center of mass of the $n$ bodies $=\sum m_{i} R_{i}=0$ identically. Define the angular momentum of system $C$ by $\Sigma m_{i} R_{i} \times R_{i} . C$ is a constant on any given solution. Let $U=\Sigma_{1<i<j<n} m_{i} m_{j} / r_{i j}$ be the potential of the $n$-body problem and $T=\sum m_{i} R_{i}^{.2} / 2=$ the kinetic energy. Then it follows from the equations of motion that $T-U$ is a constant on any solution. We shall call this constant $h$. It is well known that the angular momentum, $C$, and total energy, $h$, are conserved as any given solution passes through a binary collision.

MOMENT OF INERTIA. We define a parameter, $I=\sum m_{i} R_{i}^{2}$. If $M^{*}=\sum m_{i}$, then $I$ may be expressed in terms of the distances, $r_{i j}$, by $I=$ $\left(1 / M^{*}\right) \sum_{1 \leqslant i<j \leqslant n} m_{i} m_{j} r_{i j}^{2}$. There is a relation between the moment of inertia and the potential, namely the Lagrange-Jacobi formula,

$$
I \cdots=4 T-2 U=2 U+4 h=2 T+2 h .
$$

REMARK. It is possible to assume with no loss of generality that solutions of the $n$-body problem are defined for $-1 \leqslant t<0$ and that if a singularity occurs that it happens at $t=0$. We use $\lim _{t \rightarrow 0}$ to denote the limit from the left.

LemMA 1. Suppose $t=0$ is a singularity of the $n$-body problem. Then $\lim _{t \rightarrow 0} U(t)=\infty$.

Proof. If the lemma is false, then there exists an increasing sequence, $\left\{t_{m}\right\}$, with limit zero and a positive number, $a$, such that $U\left(t_{m}\right)<a$. Let $m_{0}=$ $\min m_{i}$ and we then have

$$
\min _{1<i<j<n} r_{i j}\left(t_{m}\right) \geqslant m_{0}^{2} / a=a^{\prime} .
$$

Also by the energy relation we have $\sum m_{i} R_{i}^{.2} / 2=U+h$, so

$$
\max \left|R_{i}\left(t_{m}\right)\right| \leqslant \sqrt{2(a+h) / m_{0}}=b .
$$

Now apply Cauchy's existence theorem to yield existence of analytic solutions in neighborhoods $\left|t-t_{m}\right|<\delta$ where $\delta$ does not depend on $m$. This implies 0 is not a singularity of this solution since $t=0$ will eventually fall inside one of these neighborhoods.

COROLlaRy 1. If $t=0$ is a singularity of the $n$-body problem, then $\lim _{t \rightarrow 0} I(t)=0, L$, or $\infty$.

Proof. From the Lagrange-Jacobi formula and the above result, $\lim _{t \rightarrow 0} I \cdot{ }^{\circ}$ $=\infty$. Therefore $I$ is positive by definition and convex near 0 , so the corollary follows.

Notation. We shall use $a_{m}, m=1,2, \ldots$, to denote positive constants. 
COROLlary 2. If $t=0$ is not a singularity due to a general collision of all particles, i.e., $\lim _{t \rightarrow 0} I(t) \neq 0$, then there exists some positive number $a_{1}$ such that near $t=0, \max _{1 \leqslant i<j \leqslant n} r_{i j}(t)>a_{1}$. This is a simple consequence of the formula for $I$ in terms of the distances, $r_{i j}$. Choose $a_{1}=\sqrt{L / 2 M^{*}}$.

LEMMA 2. Suppose $t=0$ is any singularity of the $n$-body problem. Then we can find a constant, $a_{2}$, such that $U(t) \geqslant a_{2} t^{-2 / 3}$.

Proof. The proof of this lemma consists of finding an easy inequality for $U \cdot$ and integrating it from $t$ to 0 [7]. Define $V=3 \cdot n$ vector of velocities, $V=\left(R_{1}, R_{2}, \ldots, R_{n}\right)$. Define $U^{\prime}=$ the gradient of $U$ with respect to its $3 n$ real arguments, $R_{i}, i=1, \ldots, n . U^{\prime}=\left(U_{R_{1}}, U_{R_{2}}, \ldots, U_{R_{n}}\right)$. Let $|V|,\left|U^{\prime}\right|=$ Euclidean norms of the respective vectors. By the energy relation,

$$
T=U+h=\frac{1}{2} \sum m_{i}\left(R_{i}\right)^{2}>\frac{1}{2} m_{0}|V|^{2} .
$$

Therefore

$$
|V| \leqslant\left(2 T / m_{0}\right)^{1 / 2} \leqslant b_{1} U^{1 / 2}
$$

since $T=U+h$ and $U$ tends to infinity. Now

$$
U_{R_{i}}=\Sigma^{\prime} m_{i} m_{j} R_{j i} / r_{i j}^{3} \text {. }
$$

Therefore

$$
\left|U^{\prime}\right| \leqslant\left(M^{* 2} / m_{0}^{4}\right) U^{2}=b_{2} U^{2}
$$

Since $U \cdot=U^{\prime} V$,

$$
|U \cdot| \leqslant b_{1} b_{2} U^{5 / 2}=b_{3} U^{5 / 2} .
$$

The desired inequality is

$$
\left|\left(U^{-3 / 2}\right)\right| \leqslant(3 / 2) b_{3} \text {. }
$$

We can integrate this inequality from $t$ to 0 since Lemma 1 gives $U^{-5 / 2}(S)$ tends to 0 as $S$ tends to 0 . Therefore, $\left|U^{-3 / 2}\right| \leqslant(3 / 2) b_{3}|t|$, so if $a_{2}=$ $\left(1 /(3 / 2) b_{3}\right)^{2 / 3}$, the lemma is clear.

NONCOLLISION SINGULARITY IN THE FOUR-BODY PROBLEM. In the remainder of the paper we assume that $t=0$ is a noncollision singularity of the 4-body problem.

2. In this section we begin the study of noncollision singularities. The section begins with a discussion of "cluster coordinates", a basic tool for the study of this subject. It may be seen from the formulas given in this discussion that the center of mass of an isolated cluster of particles will move with essentially rectilinear motion, and that the motion of particles in an isolated cluster will be most strongly influenced by the mutual attractions among the particles of the cluster. 
It is then inferred from Lemmas 1 and 2 that any clustering of the four masses must be of one of the two types illustrated in Figure 1. The impact of Lemmas $3-5$ is that the open time interval immediately preceding the time of the singularity may be partitioned into countably many interlocking intervals on which cluster configurations $\boldsymbol{A}$ or $\boldsymbol{B}$ are alternately in effect (see Figure 2).

A. Cluster configuration $A$ $t \in W_{j}$

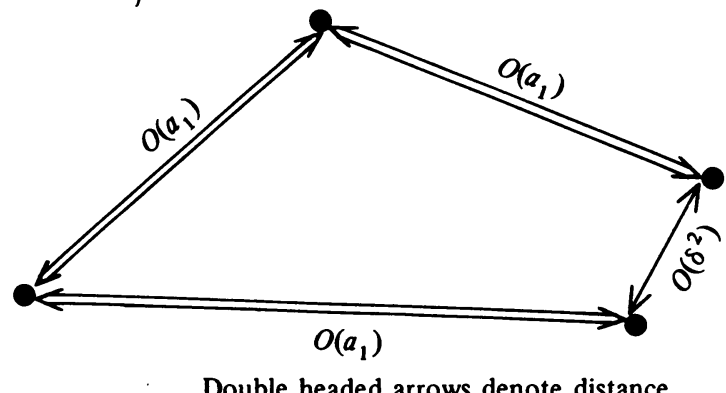

Double headed arrows denote distance

B. Cluster configuration $B$ $t \in s_{k}$

(1)

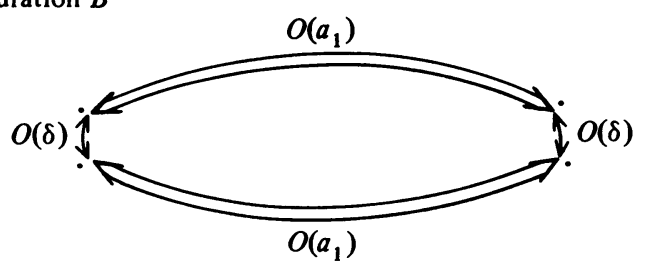

(2)

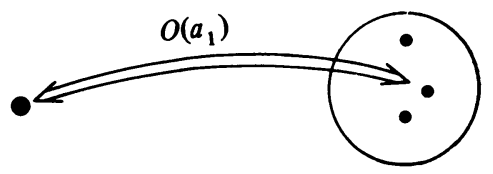

Figure 1

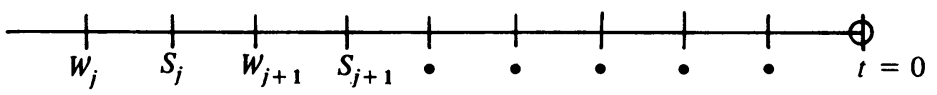

FIGURE 2

Clusters. In the study of singularities of the $n$-body problem it is sometimes useful to segregate the $n$ masses into clusters. This is done in the following 
way. Let $G_{s}, s=1, \ldots, P, P \leqslant n$, be disjoint subsets of the set $N=\{1$, $2, \ldots, n-1, n\}$ with $\cup{ }_{s=1}^{P} G_{s}=N$. The masses whose indices belong to the set $G_{s}$ are said to be in the $s$ th cluster.

FORMULAS. Let

$$
M_{s}=\sum_{i \in G_{s}} m_{i}=\text { the total mass of the } s \text { th cluster. }
$$

Let

$$
C_{s}=\frac{1}{M_{s}} \sum_{i \in G_{s}} m_{i} R_{i}
$$

$C_{s}$ is the center of mass of the $s$ th cluster. We have cluster formulas for $I$ and $C$. The first one is

$$
I=\sum_{s=1}^{P} \sum_{i \in G_{s}} m_{i}\left(R_{i}-C_{s}\right)^{2}+\sum_{s=1}^{P} M_{s} C_{s}^{2}
$$

The cluster formula for angular momentum is given by

$$
C=\sum_{s=1}^{P} \sum_{i \in G_{s}} m_{i}\left(R_{i}-C_{s}\right) \times\left(R_{i}-C_{s}\right)+\sum_{s=1}^{P} M_{s} C_{s} \times C_{s}
$$

Choosing Natural Clusters. As stated the selection of clusters may be arbitrary, but normally clusters are chosen so that particles which are close together are in the same cluster. Suppose the distances, $r_{i j}$, fall into two categories: (1) greater than $a$, (2) less than $a / 2$, for some $a$. This induces a relation on $N, i$ related to $j$ if $r_{i j}<a / 2$. By the triangle inequality, this is an equivalence relation and we can therefore use it to partition $N$ into subsets $G_{s}$. This assures that if $i \in G_{s}$ and $j \notin G_{s}$, then $r_{i j}>a$.

ESTIMATE FOR $C_{s} \cdot \cdot$. Suppose $0<r=\min \left\{r_{i j}, i \in G_{s}, j \notin G_{s}\right\}$. We have

$$
\begin{aligned}
M_{s} C_{s} & =\sum_{i \in G_{s}} \sum_{\substack{j \in G_{s} \\
j \neq i}} \frac{m_{i} m_{j} R_{j i}}{r_{i j}^{3}}+\sum_{i \in G_{s}} \sum_{j \notin G_{s}} \frac{m_{i} m_{j} R_{j i}}{r_{i j}^{3}} \\
& =\sum_{i \in G_{s}} \sum_{j \notin G_{s}} \frac{m_{i} m_{j} R_{j i}}{r_{i j}^{3}} .
\end{aligned}
$$

This follows because

$$
\sum_{i \in G_{s}} \sum_{\substack{j \in G_{s} \\ j \neq i}} F(i, j)=0 \text { if } F(i, j)=-F(j, i) .
$$

Therefore,

$$
\left|M_{s} C_{s} \cdot\right| \leqslant M^{* 2} / r^{2}
$$


Technical note. Normally, a group of particles is said to form a cluster if the mutual distances of these particles are all less than a preassigned positive number. In this paper we will define clusters with respect to a sliding parameter, $\delta$, a function of time which tends to 0 at the time of the singularity. The advantage in doing this is that trivial clusters, i.e. clusters in which small velocity changes occur, are avoided. In other words, by a careful choice of the function $\delta$, it will be possible to show that:

(a) When a particle or cluster is isolated from all other particles by a distance at least $\delta$, then the velocity of the particle or cluster is essentially constant (Lemmas 3 and 4).

(b) In any cluster of size $\delta$ or less, immense changes of velocity must occur. Lemma 5 asserts that two successive clusters of size $\delta$ or less must consist of different particles. The fact that the clusters do not immediately recur implies that a large change in velocity, and therefore a strong interaction, must occur during the time in which the particles are clustered. It can be seen in the proof of Lemma 5 that this nonrecurrence of clusters is a trivial consequence of the choice of the parameter $\delta$.

Construction of CLUSTERING INTERVALS. Let $\delta$ be a function of time defined for $t<0$ by $\delta(t)=|t|^{1 / 3}$. We restrict attention to $t$ such that $a_{2} \delta^{2}(t) \leqslant \delta(t) / 2 \leqslant a_{1} / 16$. By Lemma 1 , Corollary 2 and Lemma 2 we know that for $t$ near 0 ,

$$
\min _{i \neq j} r_{i j}<M^{* 2} \delta^{2} / a_{2}=a_{2}^{*} \delta^{2} \text { and } \max r_{i j}>a_{1} .
$$

Define $E$ to be the set of $t$ such that second smallest of $r_{i j}(t) \geqslant \delta / 2$. On connected components of $E$ pick out $I, J$ the indices of the unique pair whose distance, $r_{I J}$, is the minimum of all the distances, $r_{i j}$. Let $G_{1}=(I, J), G_{2}$ and $G_{3}$ each contain precisely one element. Let $C_{s}$ be defined from $G_{s}, s=1,2,3$. Let $C_{q s}=C_{q}-C_{s}, q=1,2,3, s=1,2,3$ and let $c_{q s}=\left|C_{q s}\right|$. Define $W$ to be the set $\left\{t \in E \mid \min _{s \neq q} c_{s q} \geqslant \delta(t)\right\}$.

In the construction thus far we have only used the assumption that the solution is not a total collapse. On the other hand, we have little information about the set $W$.

LeMma 3. Let $t$ and $\tau$ be elements of a connected component of $W$. Also let $t_{1}$ be left endpoint of this connected component. Then $\left|C_{s}^{*}(t)-C_{s}(\tau)\right|<a_{3} \delta\left(t_{1}\right)$.

Proof.

where

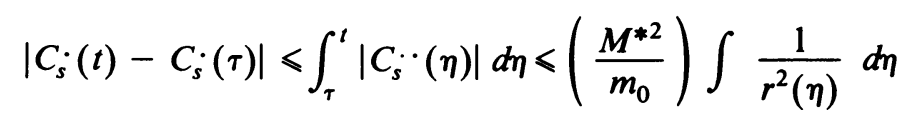

$$
r(\eta)=\min _{\substack{i \in G_{s} \\ j \notin G_{s}}} r_{i j}(\eta)>\delta(\eta)-a_{2}^{*} \delta^{2}(\eta)>\delta(\eta) / 2
$$


Then

$$
\left|C_{s}(t)-C_{s}(\tau)\right| \leqslant \frac{4 M^{* 2}}{m_{0}} \int \frac{1}{\delta^{2}(\eta)} d \eta<a_{3} \delta(\tau)<a_{3} \delta\left(t_{1}\right)
$$

since $t_{1} \leqslant \tau$.

COROLlaRY. Since $t=0$ is a noncollision singularity of the four-body problem, $t=0$ must be a limit point of $W$ complement.

Proof. If this were not the case then the clusters $G_{1}, G_{2}, G_{3}$ in effect on the last component of $W$ would have velocities $C_{\mathrm{i}}, C_{\dot{2}}, C_{\dot{3}}$ which one could integrate up to the origin. This would then give limiting values for the position vectors $R_{1}, R_{2}, R_{3}, R_{4}$ since two of the clusters are trivial and the other consists of two particles separated by distance less than $a_{2}^{*} \delta^{2}$, which tends to 0 .

LeMma 4. Let $S_{1}$ be a component of $W$ complement. Then on the interval $S_{1}$ there is a clustering arrangement consisting of two clusters $H, H^{\prime}$ with $\min _{i \in H ; j \in H^{\prime}}, r_{i j}>a_{1} / 2$ and $\max _{i, j \in H ; i, j \in H^{\prime}} r_{i j}<2 \delta$.

Notation. Let $H, H^{\prime}$ be the clustering on a component of $W$ complement. Let

$$
\begin{array}{cl}
M=\sum_{i \in H} m_{i}, & M^{\prime}=\sum_{i \in H^{\prime}} m_{i}, \\
D=\frac{1}{M} \sum_{i \in H} m_{i} R_{i}, & D^{\prime}=\frac{1}{M^{\prime}} \sum_{i \in H^{\prime}} m_{i} R_{i} .
\end{array}
$$

Corollary 1. Let $\tau<t$ be two points in $S_{1}$. Then $|D \cdot(t)-D \cdot(\tau)|<a_{4}(t-$ $\tau)$ and $\left|D^{\prime \cdot}(t)-D^{\prime} \cdot(\tau)\right| \leqslant a_{4}(t-\tau)$.

Proof. Let $a_{4}=4\left(M^{* 2} / m_{0}\right) / a_{1}^{2}$. (See proof of Lemma 3.)

COROllary 2. The point $t=0$ is a limit point of $W$.

Proof. The preceding corollary insures that $D \cdot$ and $D^{\prime \cdot}$ are integrable over components of $W$ complement. If $W$ is bounded away from 0 , then $D^{\prime}$ and $D \cdot$ are integrable and thus $D$ and $D^{\prime}$ have limits as $t$ tends to 0 . Since $i, j$ elements of $H$ or $i, j$ elements of $H^{\prime}$ imply $r_{i j}<2 \delta$, then $R_{i}$ must also have a limit at 0 .

Notation for Lemma 5. Let $S_{0}, W_{1}, S_{1}$ be components of $W$ complement, $W$, and $W$ complement which are adjacent, with $S_{0}$ to the left of $W_{1}$ to the left of $S_{1}$. Let $H_{0}, H_{0}^{\prime}$ be the clusters on $S_{0}, G_{1}, G_{2}, G_{3}$ on $W_{1}$, and $H_{1}, H_{1}^{\prime}$ on $S_{1}$.

REMARK. One of the clusters on $S_{0}$ must be the union of two of the three clusters $G_{1}, G_{2}, G_{3}$ and one of the clusters of $S_{1}$ must be the union of two of 
the clusters $G_{1}, G_{2}, G_{3}$. That is, Lemmas 3 and 4 tell us that the clusters $H, H^{\prime}$ break up and reform infinitely often near a noncollision singularity. We are now tacitly assuming that the entire system, $S_{0}, W_{1}, S_{1}$ is in some arbitrarily small fixed neighborhood of $t=0$. This is possible by the corollaries to Lemmas 3 and 4.

LemMA 5. Suppose $H_{0}=G_{s_{0}} \cup G_{p_{0}}$ and $H_{1}=G_{s_{1}} \cup G_{p_{1}}$ for integers $1 \leqslant s_{k}$ $<p_{k} \leqslant 3$ for $k=0,1$. Then $\left\{s_{0}, p_{0}\right\} \neq\left\{s_{1}, p_{1}\right\}$.

Proof. Suppose $\left\{s_{0}, p_{0}\right\}=\left\{s_{1}, p_{1}\right\}$. Since $s_{k}$ was chosen less than $p_{k}$, the above supposition implies $s_{0}=s_{1}$ and $p_{0}=p_{1}$. Then let $s=s_{1}, p=p_{1}$. Let $t_{1}, t_{2}$ be the left and right endpoints of $W_{1}$, respectively. Define $f(t)$ for $t$ in a neighborhood of $W_{1}$ by $f(t)=c_{s p}(t)-\delta(t)$. Notice that by construction $f$ must have a maximum on $W_{1}$ even if $W_{1}$ is only a point, since for $t$ immediately to the left of $t_{1}, f(t)<0$, and for $t$ immediately to the right of $t_{2}$, $f(t)<0$, and for $t \in\left[t_{1}, t_{2}\right], f(t) \geqslant 0$. Therefore there is a point, $t^{*}$, element of $W_{1}$ such that $f^{\cdot} \cdot\left(t^{*}\right) \leqslant 0$.

Calculation. Let $R(t)$ be a $C^{2}$ map of some interval into $R^{n}$. Let $r(t)=|R(t)|$ and suppose $r(t) \neq 0$. Then

$$
\begin{aligned}
r \cdot(t)= & (R(t) R \cdot(t) / r(t))=R(t) R \cdot \cdot(t) / r(t) \\
& +\left(R \cdot{ }^{2}(t) / r(t)-(R(t) R \cdot(t))^{2} / r^{3}(t)\right) \\
\geqslant & R(t) R \cdot \cdot(t) / r(t) \geqslant-|R \cdot \cdot(t)| .
\end{aligned}
$$

Since $W \subset E$; the functions $C_{s}, s=1,2,3$, are of class $C^{2}$ in a neighborhood of $W$. Apply this to the functions $C_{s p}$ and $c_{s p}$ in the following calculations:

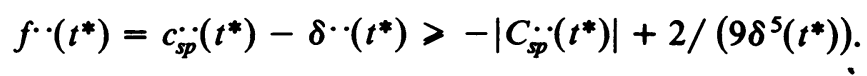

However, since $t^{*} \in W_{1}, c_{12}, c_{13}$, and $c_{23}$ satisfy $c_{s p} \geqslant \delta$ and

$$
\left|C_{s p}\right|=\left|C_{s} \cdot C_{\dot{p}} \cdot \leqslant\right| C_{s} \cdot|+| C_{p} \cdot \mid<\left(8 M^{* 2} / m_{0}\right) / \delta^{2}
$$

therefore

$$
f \cdot\left(t^{*}\right) \geqslant\left(1 / \delta^{5}\left(t^{*}\right)\right)\left(\frac{2}{9}-\left(8 M^{* 2} / m_{0}\right) \delta^{3}\left(t^{*}\right)\right)>0
$$

as $\delta(t)$ tends to 0 as $t$ tends to 0 . Therefore $f$ is strictly convex on $W_{1}$ and this is the contradiction.

COROLlaRY (1). $t_{2}-t_{1} \geqslant\left(a_{1} /\left(2 \max _{t \in W_{1} ; s \in\{1,2,3\}}\left|C_{s}\right|\right)\right)$.

This is clear since one of the centers, $C_{s}$, must move in some fashion from one location to another during $W_{1}$ and these locations are separated by at least $a_{1} / 2$.

COROLlaRY (2). 0 is the only limit point of the boundary of $W$. 
Proof. From Lemma 5 and the construction of the clusters $G_{s}$, it is clear that any limit point of the boundary of $W$ must be a point in time at which some of the coordinate functions $R_{i}$ do not have limits. This contradicts the assumption that the solution $R_{i}, R_{i}, i=1,2,3,4$, has no singularity for $t<0$.

REMARKS. Since the boundary of $W$ does not accumulate at any point $t_{0}<0$, we may enumerate the components of $W$ in ascending order. $W_{k+1}$ is the left-most component of $W$ which lies to the right of $W_{k}$.

Similarly, let $S_{k}$ be the components of the complement of $W$ enumerated such that $S_{k}$ lies between $W_{k}$ and $W_{k+1}$.

Notation. Let $H_{k}$ be one of the two natural clusters on $S_{k}$. Let $G_{1 k}, G_{2 k}, G_{3 k}$ be the three clusters formed on $W_{k}$. Where the meaning is clear, the subscript $k$ will be suppressed. Let $M_{k}=\sum_{i \in H_{k}} m_{i}$. Let $D_{k}=\left(1 / M_{k}\right) \sum_{i \in H_{k}} m_{i} R_{i}=$ center of mass of $H_{k}$. Let $T_{1 k}, T_{2 k}=$ left and right endpoints of $W_{k}$.

3. This section completes the basic material needed to state and prove Theorem 1. Lemma 7 gives us a crude picture of how the overall size of the system must increase without bound at the time of a noncollision singularity (see Figure 3).

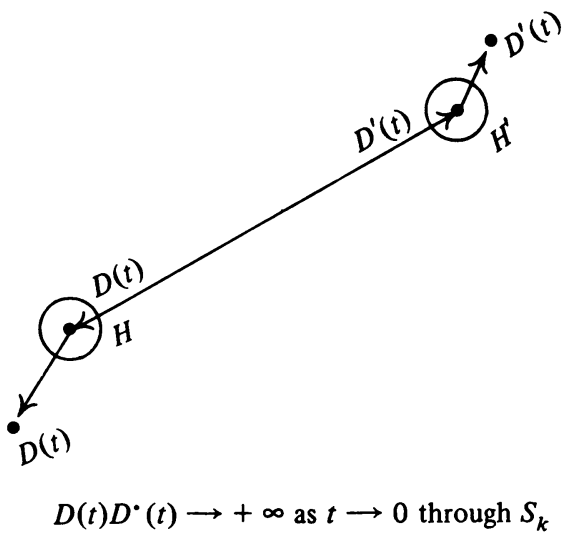

FIGURE 3

LEMMA 6. Suppose $t=0$ is a noncollision singularity of the $n$-body problem, then $\lim _{t \rightarrow 0} I \cdot(t)=\infty$.

Proof. By Lemma 1 and the fact that $t$ is chosen to increase toward $0^{-}, I^{\text {. }}$ is increasing for $t$ near $0^{-}$. If Lemma 6 is false, $I \cdot$ approaches a limit at $0^{-}$. Then by the Lagrange-Jacobi relationship, $T$ is integrable. So the functions $R_{i}(t)$ are actually square integrable and thus integrable near 0 . This contradicts $t=0$ is a noncollision singularity.

LEMMA 7. The limit of $D(t) D \cdot(t)=\infty$ as $t$ tends to 0 through $S_{k}$. 
Proof. The proof of this lemma uses Lemmas 5 and 6. Let $H^{\prime}$ be the other cluster on $S$ and $M^{\prime}$ and $D^{\prime}$ defined analogously with $M$ and $D$. Note $M^{\prime}=M^{*}-M$ and $D^{\prime}=-M D / M^{\prime}$.

By the cluster formula for $I$, we have

$$
I=M D^{2}+M^{\prime} D^{\prime 2}+I^{*}+I^{* \prime}
$$

where

$$
I^{*}=\sum_{i \in H} m_{i}\left(R_{i}-D\right)^{2} \text { and } I^{* \prime}=\sum_{i \in H^{\prime}} m_{i}\left(R_{i}-D^{\prime}\right)^{2}
$$

Note

$$
I^{*}=\left(\frac{1}{M}\right) \sum_{\substack{i, j \in H \\ i<j}} m_{i} m_{j} r_{i j}^{2}
$$

and similarly for $I^{* \prime}$. We seek a point $\tau_{k} \in W_{k}$ near $S_{k}$ such that

$$
\left(I^{*}\left(\tau_{k}\right)+I^{* \prime}\left(\tau_{k}\right)\right)^{\cdot} \leqslant 0 .
$$

Let

$$
\eta_{k}=\sup \left\{t \in W_{k} \mid \max _{\substack{i, j \in H \\ i, j \in H^{\prime}}} r_{i j}>\frac{8 M^{*}}{m_{0}} \delta\left(T_{2 k}\right)\right\} .
$$

$\eta_{k}$ is well defined since by Lemma 5 the clusters $G_{s}$ and $G_{p}$ which coalesce to form $H$ or $H^{\prime}$ on $S_{k}$ do not comprise $H_{k-1}$ or $H_{k-1}^{\prime}$. Therefore the set of which $\eta_{k}$ is the supremum cannot be empty. By the continuity of $r_{i j}, r_{i j}\left(\eta_{k}\right)=$ $8 M^{*} \delta\left(T_{2 k}\right) / m_{0}$ for some $(i, j)$ belonging to $H$ or $H^{\prime}$. This implies $I^{*}\left(\eta_{k}\right)+$ $I^{* \prime}\left(\eta_{k}\right)>64 M^{*} \delta^{2}\left(T_{2 k}\right)$. Conversely on $S_{k}, i \in H$ implies $\left|R_{i}-D\right|<2 \delta$ and $j \in H^{\prime}$ implies $\left|R_{j}-D^{\prime}\right|<2 \delta$. Therefore

$$
I^{*}\left(T_{2 k}\right)+I^{* \prime}\left(T_{2 k}\right) \leqslant 4 M^{*} \delta^{2}\left(T_{2 k}\right)<I^{*}\left(\eta_{k}\right)+I^{* \prime}\left(\eta_{k}\right),
$$

which with the mean value theorem implies the existence of $\tau_{k} \in\left(\eta_{k}, T_{2 k}\right)$ for which (1) is true. To show that (1) implies the assertion of the lemma, write

$$
\begin{aligned}
I \cdot\left(\tau_{k}\right) & \leqslant\left(M D^{2}+M^{\prime} D^{\prime 2}\right)^{\cdot}=\left(M D^{2}+M^{\prime}\left(-M D / M^{\prime}\right)^{2}\right)^{\cdot} \\
& =M\left(1+M / M^{\prime}\right) D^{2 \cdot}=2 M\left(1+M / M^{\prime}\right) D D^{.}
\end{aligned}
$$

which, therefore, tends to infinity as $k$ tends to infinity.

To prove the lemma, it now suffices to prove $\left(D D^{*}\right)^{\cdot}$ is bounded below on $\left(\tau_{k}, T_{1(k+1)}\right)$. To do this, write

$$
\left(D D^{\cdot}\right)^{\cdot}=(D \cdot)^{2}+D D \cdot \geqslant-|D||D \cdot \cdot \geqslant-| D \mid M^{* 2} m_{0}^{-1} / r^{2}
$$

where $r(t)=\min _{i \in H ; j \in H^{\prime}} r_{i j}(t)$. Observe for $\eta_{k} \leqslant t<T_{1(k+1)}$,

$$
r>\left|D-D^{\prime}\right|-16 M^{*} m_{0}^{-1} \delta\left(T_{2 k}\right)
$$


by definition of $\eta_{k}$. Since $M D+M^{\prime} D^{\prime}=0,\left|D-D^{\prime}\right|=|D|+\left|D^{\prime}\right|>|D|$. Therefore $r>|D| / 2$. By Corollary 2 of Lemma 1 , one distance $r_{i j}$ is greater than $a_{1}$, so again by definition of $\eta_{k}$,

$$
r>a_{1}-16\left(M^{*} / m_{0}\right) \delta\left(T_{2 k}\right)>a_{1} / 2 \text {. }
$$

Thus $(D D \cdot) \cdot>-4\left(M^{* 2} / m_{0} a_{1}\right)$. This proves the lemma.

Summary OF Cluster Construction. We have constructed a sequence of intervals $W_{k}, S_{k}$ which accumulate at 0 . On $W_{k}$ there are three clusters, $G_{1}$, $G_{2}, G_{3}$, all separated by at least $\delta(t) / 2$. It was shown that for $t, \tau \in W_{k}$ that $\left|C_{s}(t)-C_{s}(\tau)\right|<a_{3} \delta\left(T_{1 k}\right)$. On the interval $S_{k}$ we have only two clusters $H$, $H^{\prime}$. We have shown that one of these clusters is $G_{s} \cup G_{p}$ for $1<s<p<3$. We have also shown that $\min \left\{r_{i j}(t) \mid i \in H, j \in H^{\prime}, t \in S_{k}\right\}>a_{1} / 2$. Therefore for $t, \tau \in S_{k},|D \cdot(t)-D \cdot(\tau)|<a_{4}|t-\tau|$.

We also know that one of the two clusters $\left\{H_{k-1}, H_{k-1}^{\prime}\right\}$ on $S_{k-1}$ is $G_{s_{k}^{\prime}} \cup G_{p_{k}^{\prime}}$ for $1<s^{\prime}<p^{\prime}<3$. We have from Lemma 5 that $\left\{s^{\prime}, p^{\prime}\right\} \neq\{s, p\}$. Therefore

$$
\begin{array}{cr}
c_{s^{\prime} p^{\prime}}\left(T_{1 k}\right)=\delta\left(T_{1 k}\right), & c_{s^{\prime} p^{\prime}}\left(T_{2 k}\right)>a_{1} / 2, \\
c_{s p}\left(T_{1 k}\right)>a_{1} / 2, & c_{s p}\left(T_{2 k}\right)=\delta\left(T_{2 k}\right) .
\end{array}
$$

\section{THEOREM 1}

Discussion. It would be reasonable to suppose that a clustering of particles during some time interval, for example $H, H^{\prime}$ on $s_{k}$, would represent a very strong interaction among the particles of each cluster since these particles must, by definition, be in extremely close proximity. On the other hand, the degree to which particles of a cluster interact with each other is governed by the initial velocities of the individual particles as well as by the size of the cluster. Theorem 1 is proved by showing that each interaction must be sufficiently strong to produce radical changes in the subclusters $G_{s}$ and $G_{p}$ during the time interval, $s_{k}$. The assertions of the theorem may be interpreted to state that the clusters $H, H^{\prime}$ must be in a sense near collisions.

Notation. Let $H=G_{s} \cup G_{p}$ (see above). Define $M, D, Z$ from $H$ as before and define $H^{\prime}, M^{\prime}, D^{\prime}$ as in Lemma 7. Let

$$
Z(t)=\sum m_{i}\left(R_{i}-D\right) \times\left(R_{i}-D\right)
$$

summed over $i \in H$ and let

$$
Z^{\prime}=\sum_{i \in H^{\prime}} m_{i}\left(R_{i}-D^{\prime}\right) \times\left(R_{i}-D^{\prime}\right) .
$$

$Z, Z^{\prime}$, are defined for $t$ near each $S_{k}$.

THEOREM 1. The limits of $Z(t)$ and $Z^{\prime}(t)$ are zero as $t$ tends to 0 through $S_{k}$. 
Remarks. The proof of Theorem 1 will be done by contradiction, but we will not assume Theorem 1 is false yet.

4. In this section we initiate the proof of Theorem 1. Lemma 8 asserts that, for the purpose of this analysis, the angular momentum of the clusters $H, H^{\prime}$ remains essentially constant during the time interval, $S_{k}$. Lemmas 9 and 10 reduce the problem of analyzing the angular momentum of the clusters $H, H^{\prime}$ to that of analyzing the asymptotic behavior of a vector valued function, $R$, and its first derivative, $V$. See Figure 4.

A.

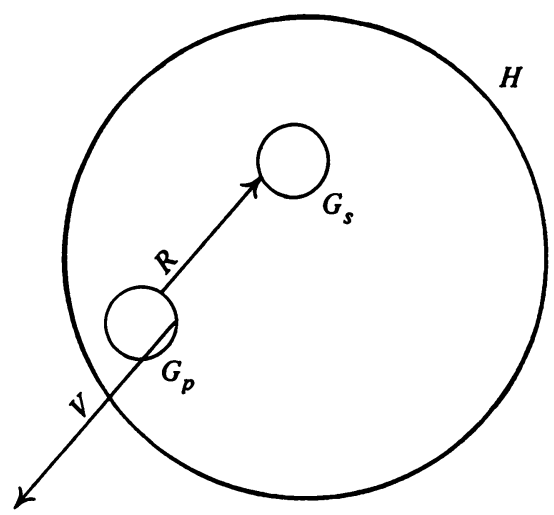

$Z \rightarrow 0$ implies $(R \times V) \rightarrow 0$

B.

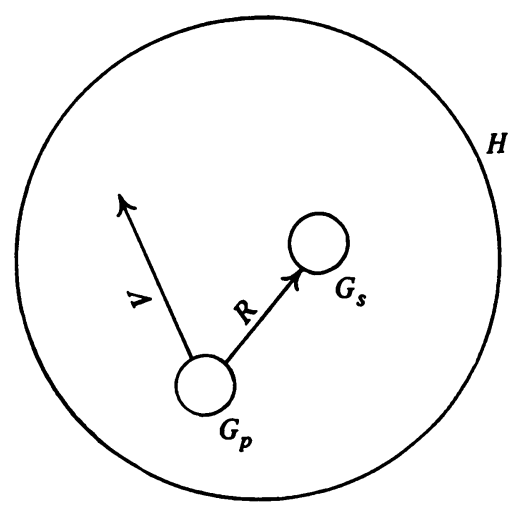

$|Z|$ bounded away from zero implies $|(R \times V)|$ bounded away from zero

Figure 4

LEMMA 8. Suppose $\zeta_{i}$ satisfy

$$
\zeta_{i} \cdot=\sum_{i \neq j} \mu_{j}\left(\zeta_{j}-\zeta_{i}\right) /\left|\zeta_{j}-\zeta_{i}\right|^{3}+P_{i}
$$


where $P_{i}$ are bounded functions, $\mu_{j}$ are positive numbers, and $i, j$ belong to some finite set of integers $G$. Then if $Y=\Sigma_{i \in G} \mu_{i} \zeta_{i} \times \zeta_{i}$ we have

$$
|Y \cdot|<\sum_{i \in G}\left|\mu_{i} \zeta_{i}\right|\left|P_{i}\right|
$$

Proof. Compute

$$
\begin{aligned}
Y \cdot & =\sum_{i \in G} \mu_{i} \zeta_{i} \times \zeta_{i} \cdot \\
& =\sum_{\substack{i, j \in G \\
i \neq j}} \mu_{i} \mu_{j} \zeta_{i} \times \zeta_{j} /\left|\zeta_{j}-\zeta_{i}\right|^{3}+\sum_{i \in G} \mu_{i} \zeta_{i} \times P_{i} \\
& =\sum_{i \in G} \mu_{i} \zeta_{i} \times P_{i},
\end{aligned}
$$

since the terms in the double sum are antisymmetric in $i, j$. Therefore $|Y \cdot|<\Sigma_{i \in G}\left|\mu_{i} \zeta_{i}\right|\left|P_{i}\right|$.

Corollary. For $t \in S_{k}$, (1) $|Z \cdot(t)| \leqslant 8 M^{* 2} \delta(t) / a_{1}^{2}$. Also, (2) $\left|Z^{\prime} \cdot(t)\right| \leqslant$ $8 M^{* 2} \delta(t) / a_{1}^{2}$.

Proof. To get (1), apply Lemma 8, $G=H, \zeta_{i}=R_{i}-D, P_{i}=$ $\left(\Sigma_{j \in H^{\prime}} m_{j} R_{j i} / r_{i j}^{3}\right)-D \cdot, \mu_{i}=m_{i}$. For (2) $G=H^{\prime}, \zeta_{i}=R_{i}-D^{\prime}, P_{i}=$ $\left(\sum_{j \in H^{\prime}} m_{j} R_{j i} / r_{i j}^{3}\right)-D \cdots, \mu_{i}=m_{i}$.

REMARKS. We will deal with a number of velocities, $R_{i}, i=1, \ldots, 4, C_{s}$, $s=1,2,3$, and relative velocities, $R_{i j}$, $C_{s p}$, etc. All of these velocities are finite linear combinations of the four velocities $R_{i}$. To simplify notation we find a master constant, $a_{5}^{\prime}$, such that each of these finite linear combinations, $V$, satisfies

$$
|V|<a_{5}^{\prime} T^{1 / 2}=a_{5}^{\prime}(U+h)^{1 / 2}<a_{5} U^{1 / 2} .
$$

Notation. $d(t)=\min \left\{r_{i j}(t) \mid 1<i<j<4\right\}$. For Lemma 9, let $M_{g}=$ $\sum_{i \in G} m_{i}, C_{g}=\left(1 / M_{g}\right) \sum_{i \in G} m_{i} R_{i}$ and $Z_{g}=\sum_{i \in G} m_{i}\left(R_{i}-C_{g}\right) \times\left(R_{i}-C_{g}\right) \cdot$ for $G$ some cluster.

LEMMA 9. Let $G$ be a cluster with the property that $\max \left\{r_{i j}(t) \mid i, j \in G\right\}<$ $a_{6} d(t)$. Then $\left|Z_{g}\right|<M_{g} M^{* 2} a_{5} a_{6} U^{-1 / 2}$.

Proof. Trivially,

$$
\begin{aligned}
\left|Z_{g}\right| & <\sum_{i \in G} m_{i}\left|R_{i}-C_{g}\right|\left|R_{i}-C_{\dot{g}}\right| \\
& <M g \max _{i \in G}\left|R_{i}-C_{g}\right| \max _{j \in G}\left|R_{j}-C_{\dot{g}}\right| .
\end{aligned}
$$

Since $C_{g}$ is a convex combination of $R_{i}, i \in G$, then

$$
\max _{i \in G}\left|R_{i}-C_{8}\right|<\max _{i, j \in G} r_{i j}<a_{6} d<a_{6} M^{* 2} / U \text {. }
$$


Also, by the remarks in which $a_{5}$ was defined, $\max _{j \in G}\left|R_{i}-C_{\dot{g}}\right|<a_{5} U^{1 / 2}$. The lemma is clear.

Corollary 1. Suppose there exist a subsequence $k_{q}$, a positive number $C^{*}$ and points $T_{q} \in S_{k_{q}}$ such that $\left|Z\left(T_{q}\right)\right|>C^{*}$. Then the minimum distance, $r_{I J}$, is unique on $S_{k_{q}}$.

Proof. By the corollary to Lemma $8,|Z|$ tends to 0 as $t$ approaches 0 through $S_{k}$, and since length of $S_{k}$ also approaches 0 , it is certainly true that for large $q,|Z(t)|>\left|Z\left(t_{q}\right)\right| / 2>C^{*} / 2, t \in S_{k_{q}}$. Suppose $T_{q}^{\prime} \in S_{k_{q}}$ and there are two distances equal to $d\left(T_{q}^{\prime}\right)=$ minimum distances at $T_{q}^{\prime}$. Then it must be true that $i, j \in H$ or $i, j \in H^{\prime}$ implies $r_{i j}\left(T_{q}^{\prime}\right)<2 d\left(T_{q}^{\prime}\right)$; therefore, by Lemma 9, $Z\left(T_{q}^{\prime}\right), Z^{\prime}\left(T_{q}^{\prime}\right)$ tend to 0 . This is a contradiction.

Notation. $p^{\prime}=6-s-p$. So $\{1,2,3\}=\left\{s, p, p^{\prime}\right\}$.

Corollary 2. Limit of $Z(t)=0$ as $t$ tends to 0 through $S_{k}$.

Proof. We will prove that $Z^{\prime}\left(T_{2 k}\right)$ tends to 0 . This plus the corollary of Lemma 8 will prove Corollary 2 . We have $H^{\prime}=G_{p^{\prime}}$. At $T_{2 k}, G_{1}, G_{2}, G_{3}$, each contains a single particle or two particles whose distance is the minimum. Lemma 9 then assures $Z^{\prime}\left(T_{2 k}\right)$ tends to 0 .

REMARKS. Before we proceed to Lemma 10, we should recall the cluster formula for angular momentum and that its proof does not require the center of mass of the system to be fixed at the origin. This allows us to use the formula in a slightly more general setting where the system which we wish to consider is a cluster which is the union of smaller clusters. Precisely, we view $H$ as a system composed of two clusters, $G_{s}, G_{p}$, and we write

$$
Z=M_{s}\left(C_{s}-D\right) \times\left(C_{s}-D\right)^{\cdot}+M_{p}\left(C_{p}-D\right) \times\left(C_{p}-D\right)^{\cdot}+Z_{g s}+Z_{g p}
$$

where $Z_{g s}$ is the angular momentum of $G_{s}$ with respect to its center of mass, $C_{s}$, and likewise for $Z_{g p}$.

Notation. Let $R(t)$ be defined for $t$ in a neighborhood of $S_{k}$ by $R(t)=$ $C_{s}(t)-C_{p}(t)$. Let $V(t)=R \cdot(t), r(t)=|R(t)|$, and $v(t)=|V(t)|$.

REMARK. The next lemma consists of two parts with separate hypotheses and conclusions. These ideas are grouped into one lemma because the methods of proof are nearly identical.

LemMa 10. (1) Suppose the limit of $Z(t)=0$ as $t$ tends to 0 through $S_{k}$. Then define $T_{k}=\inf \left\{t \in S_{k} \mid r(t)<1 / v(t)\right\}$. If this set is empty, then let $T_{k}=$ $T_{1(k+1)}$, the right endpoint of $S_{k}$. Then the minimum distance, $r_{I J}$, is unique on $T_{2 k}<t<T_{k}$ and the limit of $|R \times V|=0$ as $t$ tends to 0 through $\left[T_{2 k}, T_{k}\right]$.

(2) Suppose hypothesis of (1) is false. Then there is a positive number, $c$, and a subsequence, $k_{q}$, such that for $t \in S_{k_{q}},|R \times V|>c$. 
Proof. Let $\alpha=\left(1 /\left(1+M_{s} / M_{p}\right)\right)$. Then $\left(C_{s}-D\right)=\alpha R$. Let $\beta=(-1 /(1$ $\left.\left.+M_{p} / M_{s}\right)\right)$. Then $\left(C_{p}-D\right)=\beta R$. Now if $\gamma=M_{s} \alpha^{2}+M_{p} \beta^{2}$ then

$$
\gamma R \times V=M_{s}\left(C_{s}-D\right) \times\left(C_{s}-D\right)^{\cdot}+M_{p}\left(C_{p}-D\right) \times\left(C_{p}-D\right) .
$$

It is clear that we may find positive numbers, $a_{7}, a_{8}$, such that $a_{7}<\gamma<a_{8}$. Therefore

$$
\begin{aligned}
a_{7}|R \times V| & <\left|M_{s}\left(C_{s}-D\right) \times\left(C_{s}-D\right)^{\circ}+M_{p}\left(C_{p}-D\right) \times\left(C_{p}-D\right)^{\circ}\right| \\
& <a_{8}|R \times V| .
\end{aligned}
$$

(1) By supposition,

$$
r(t) \geqslant 1 / v(t)>1 /\left(a_{5} U^{1 / 2}(t)\right)>\left(1 / a_{5}\right) d^{1 / 2}(t) / M^{*},
$$

for $t \in\left[T_{2 k}, T_{k}\right.$ ]. By Lemma 4 all distances $r_{i j}, i, j \in H$, are restricted to be less than $2 \delta$, which tends to 0 . Thus the previous inequality assures that $r(t)$ is much greater than $d(t)$. Therefore the minimum distance, $r_{I J}$, is unique on $\left[T_{2 k}, T_{k}\right]$. By our choice of labeling, on $W_{k}, G_{1}=\{I, J\}, r_{I J}=d$. It therefore follows that $G_{1}$ must be $\{I, J\}$ on $\left[T_{2 k}, T_{k}\right]$ and thus by Lemma $9, Z_{g_{1}}$ tends to 0 as $t$ tends to 0 through $\left[T_{2 k}, T_{k}\right.$ ]. $Z_{g_{2}}, Z_{g_{3}}$ are identically 0 since $G_{2}$ and $G_{3}$ contain one element. Therefore from this, the modified cluster formula for angular momentum and (a), (1) is clear.

To prove (2) in the same way, all that is necessary is to use Corollary 1 of Lemma 9 to show that the minimum distance is unique on $S_{k_{q}}$ for some appropriately chosen subsequence, $k_{q}$. Assume hypothesis of (1) is false; therefore there is a subsequence, $k_{q}$, and a positive number, $c^{*}$, and points $t_{q} \in S_{k_{q}}$ such that $\left|Z\left(t_{q}\right)\right|>c^{*}$. Corollary 1 of Lemma 9 tells us that the minimum distance is unique on $S_{k_{q}}$. As before, this minimum distance $=r_{I J}$ and $G_{1}=\{I, J\}$. Therefore $Z_{g_{1}}, Z_{g_{2}}, Z_{g_{3}}$ tend to 0 on $S_{k_{q}}$. Corollary to Lemma 8 assures for $t \in S_{k_{q}},|Z(t)|>\left|Z\left(t_{q}\right) / 2\right|>c^{*} / 2$. This, (a), and the modified cluster formula for angular momentum yield the assertion of (2) if $c=$ $c^{*} / 2 a_{8}$.

5. In this section we begin with the assumption that Theorem 1 is false. Lemma 11 shows that the function $R(t)$ on the interval $S_{k}$ satisfies a system of ordinary differential equations of the form of the two-body problem with a singular perturbation. Lemmas 12 and 13 show that the asymptotic solution of this system amounts to $R \approx r_{k}+v_{k} t$, for $t \in S_{k}$. Lemmas 14, 15, and their corollaries then show that all cluster velocities, $c_{i}, i=1,2,3$, undergo virtually no relative change on the time interval $S_{k}$.

Summary. Suppose Theorem 1 is false. Note that we need only assume that $H$ have positive definite angular momentum on account of Corollary 2 of Lemma 9. This assumption is in effect through the remainder of the proof of Theorem 1. 
We now catalogue what we know in light of this assumption. We have a subsequence, $k_{q}$, and a positive number, $c$, such that $t \in S_{k_{q}}$ implies $|R \times V|$ $>c$. The clustering, $G_{1}, G_{2}, G_{3}$, is natural on the interval $\left[T_{1 k_{q}}, T_{2 k_{q}+1}\right.$ ], i.e., the minimum distance is unique, and the clusters are separated on this interval. Quantitatively, on $W_{k_{q}} \cup W_{k_{q}+1}, \min \left(c_{12}, c_{13}, c_{23}\right) \geqslant \delta$ and on $S_{k_{q}}$, $\min \left(c_{s p^{\prime}}, c_{p p^{\prime}}\right)>a_{1} / 2$. Since $|R \times V|>c$, we have

$$
r>c / v>c /\left(a_{5} U^{1 / 2}\right)>\left(c / a_{5} M^{*}\right) d^{1 / 2}=a_{9} d^{1 / 2} .
$$

Notation. Let $S_{q}=S_{k_{q}}$ and $t_{q}$ and $t_{q}^{\prime}$ be the left and right endpoints of $S_{q}$, not to be confused with their previous usage in Lemmas 9 and 10. Also set $\left(\tau_{q}, \tau_{q}^{\prime}\right)=\left(T_{1 k_{q}}, T_{2\left(k_{q}+1\right)}\right)$, respectively. Assume by proper labeling that $c_{s p}\left(\tau_{q}\right)$ $=\delta\left(\tau_{q}\right)$ (to do this we must drop the convention that $G_{1}$ contains two elements), i.e., $H_{k_{q}-1}$ or $H_{k_{q}-1}^{\prime}=G_{s} \cup G_{p^{\prime}}$. By construction of the intervals $S_{k}$ and the assumption of nonzero angular momentum, we know for $t$ in $S_{q}$, $c_{s p}(t)=r(t)<\delta(t)$ with equality at $t_{q}$ and $t_{q}^{\prime}$.

We now seek to use this information to obtain an equation for $R(t)$ on $S_{q}$ and then to asymptotically solve the equation. Let $g(R)=-R / r^{3}$ for $r>0$.

LEMma 11. For $t \in S_{q}, R(t)$ satisfies $R \cdot=\mu_{q} g(R)+f(t) / r$ where $\mu_{q}$ is a positive constant and $|f(t)|$ is bounded.

Proof. Let $B_{1}, B_{2}$, be two generic bounded vector valued functions on $S_{q}$ and write

$$
C_{s} \cdot=\frac{1}{M_{s}} \sum_{\substack{i \in G_{s} \\ j \in G_{p}}} \frac{m_{i} m_{j}\left(R_{j i}\right)}{r_{i j}^{3}}+B_{1}(t)
$$

where $B_{1}$ covers the contributions to $C_{s} \cdot$ from the particles in $G_{p^{\prime \prime}}$ Also,

$$
C_{p} \cdot \frac{1}{M_{p}} \sum_{\substack{i \in G_{s} \\ j \in G_{p}}} \frac{m_{i} m_{j} R_{i j}}{r_{i j}^{3}}+B_{2} .
$$

Combining the two equations yields

$$
\begin{aligned}
R \cdot & =C_{s} \cdot C_{p} \cdot \\
& =\left(\frac{1}{M_{s}}+\frac{1}{M_{p}}\right) \sum_{\substack{i \in G_{s} \\
j \in G_{p}}} \frac{m_{i} m_{j} R_{j i}}{r_{i j}^{3}}+B_{1}-B_{2} \\
& =\frac{M_{s}+M_{p}}{M_{s} M_{p}} \sum_{\substack{i \in G_{s} \\
j \in G_{p}}} \frac{m_{i} m_{j} R_{j i}}{r_{i j}^{3}}+B_{1}-B_{2} .
\end{aligned}
$$

There are at most two pairs of indices, $i j$, which satisfy $i \in G_{s}, j \in G_{p}$ since 
only one of the $G$ clusters contains more than one element and it contains two elements.

Let $\eta_{1}=R_{i j}$ and $\mu^{1}=m_{i} m_{j}$ for one choice of the indices $i j$, and for the other choice $\eta_{2}=R_{i j}$ and $\mu^{2}=m_{i} m_{j}$. Then the equation for $R$ reads

$$
R \cdot=\left(\left(M_{s}+M_{p}\right) / M_{s} M_{p}\right)\left(\mu^{1} g\left(\eta_{1}\right)+\mu^{2} g\left(\eta_{2}\right)\right)+B_{1}-B_{2} \text {. }
$$

Cases. If both $G_{s}$ and $G_{p}$ contain a single element then $\eta_{1}=\eta_{2}=R$, and the above equation for $R$ is better than the one advertised in the statement of the lemma. If one of the clusters, $G_{s}$ or $G_{p}$, contains two elements, they must be separated by the minimum distance, $d(t)$, and $C_{s}$ or $C_{p}$, respectively, must lie on a line between these two particles. Therefore,

$$
\left|\eta_{K}-R\right|<d(t)<\left(1 / a_{9}^{2}\right) r^{2}(t), \quad K=1,2 .
$$

Let $g^{\prime}(R)$ be the $3 \times 3$ matrix whose rows are the gradients of the components of $g$. It is a simple calculation to verify $\left|g^{\prime}(R)\right|<a_{10}^{\prime} / r^{3}$. Let $f_{K}=g\left(\eta_{K}\right)-g(R), K=1,2$. Then by the mean value theorem

$$
\begin{aligned}
\left|f_{K}\right| & \leqslant \max _{0<\gamma<1}\left|g^{\prime}\left(R+\gamma\left(\eta_{K}-R\right)\right)\right|\left|\eta_{K}-R\right| \\
& \leqslant\left(a_{10}^{\prime} /\left(r-\left(1 / a_{9}^{2}\right) r^{2}\right)^{3}\right)\left(1 / a_{9}^{2}\right) r^{2}<a_{10}^{\prime \prime} / r .
\end{aligned}
$$

The last step is justified since

$$
\left|R-\gamma\left(\eta_{K}-R\right)\right|>r-\gamma\left|\eta_{K}-R\right|>r-\left(1 / a_{9}^{2}\right) r^{2}>r / 2 .
$$

Now rewrite equation for $R$ in terms of $f_{K}$,

$$
R \cdot=\frac{M_{s}+M_{p}}{M_{s} M_{p}}\left(\left(\mu^{1}+\mu^{2}\right) g(R)+\sum_{K=1}^{2} \mu^{K} f_{K}\right)+B_{1}-B_{2} .
$$

The proof is now complete. Let

$$
\begin{gathered}
\mu_{q}=\left(\left(M_{s}+M_{p}\right) / M_{s} M_{p}\right)\left(\mu^{1}+\mu^{2}\right) ; \quad a_{10}=\left(M^{*} / m_{0}\right)^{2} a_{10}^{\prime \prime} ; \\
f(t)=r\left(M_{s}+M_{p}\right)\left(M_{s} M_{p}\right)^{-1} \sum_{K=1}^{2} \mu^{K} f_{K} .
\end{gathered}
$$

DEFINITION. Let $J(t)=\frac{1}{2} r^{2}(t)$.

$$
\begin{aligned}
J \cdot & =R V J \cdot=v^{2}+R V \cdot \geqslant v^{2}-\mu_{q} / r-|f| \\
& \geqslant v^{2}-v / c-|f| .
\end{aligned}
$$

Therefore, since $v>c / r>c / \delta$ which tends to infinity, $J \cdot \geqslant v^{2} / 2$.

LEMMA 12. We can write for $t \in S_{q}, v(t)=v\left(t_{q}\right)+v^{*}(t)$, where $v^{*}(t) / v\left(t_{q}\right)$ tends to 0 as $t$ tends to 0 through $S_{q}$. 
Proof. By definition of $J$ and the calculation of its derivatives, it follows that for large $q, J$ is convex on $S_{q}$. Therefore $J$ may change sign no more than once on $S_{q}$. Let $t_{q}^{*}$ be this point if such a point occurs in $S_{q}$. If no such point exists, $t_{q}^{*}=t_{q}^{\prime}$. Since $r\left(t_{q}\right) \leqslant \delta \cdot\left(t_{q}\right)<0, J \cdot<0$ for $t_{q}<t<t_{q}^{*}$. Write the equation for $R$ in the form $V \cdot=\mu_{q} g(R)+f(t) / r$. Dot both sides with $V$ to yield

$$
V V \cdot=\frac{1}{2} v^{2}=-\mu_{q} J \cdot\left(r^{2}(2 J)^{1 / 2}\right)+f(t) V / r
$$

Thus

$$
\begin{aligned}
\left|\frac{1}{2} v^{2}\right| & =\left|-\mu_{q} J \cdot\left(2^{1 / 2} r^{2} J^{1 / 2}\right)+f(t) V / r\right| \\
& <\left|\mu_{q} J \cdot /\left(2^{1 / 2} r^{2} J^{1 / 2}\right)\right|+|f(t) V / r| \\
& \leqslant\left|\mu_{q} J \cdot /\left(2^{1 / 2} r^{2} J^{1 / 2}\right)\right|+a_{10} v / r \\
& \leqslant\left(\mu_{q} / c^{2}\right) v^{2} \mid J \cdot /\left(2^{1 / 2} J^{1 / 2}\right)+\left(a_{10} / c\right) v^{2} .
\end{aligned}
$$

Divide the inequality by $\frac{1}{2} v^{2}$ and get

$$
\left|\frac{1}{2} v^{2} \cdot\right| /\left(\frac{1}{2} v^{2}\right)=\left|\log \left(\frac{1}{2} v^{2}\right)\right| \leqslant \mu_{q}|J \cdot|\left(2^{1 / 2} / c^{2} J^{1 / 2}\right)+\left(2 a_{10} / c\right) .
$$

Integrate both sides of the inequality to obtain

$$
\begin{aligned}
& \left|\log \left(v^{2}(t) / v^{2}\left(t_{q}\right)\right)\right| \\
& \quad \leqslant \sqrt{2}\left(\mu_{q} / c^{2}\right)\left(2 J^{1 / 2}\left(t_{q}\right)-4 J^{1 / 2}\left(t_{q}^{*}\right)+2 J^{1 / 2}\left(t_{q}^{\prime}\right)\right)+2\left(a_{10} / c\right)\left(t-t_{q}\right) \\
& \quad<a_{11} \delta\left(t_{q}\right)
\end{aligned}
$$

where we use $r(t)<\delta(t), t \in S_{q}$, the definition of $J, \delta$ is decreasing, and $\left(t-t_{q}\right)<\left|t_{q}\right|=\delta^{3}\left(t_{q}\right)$. This proves the lemma.

We have just established that $v(t)$ remains essentially constant with respect to its starting value on the intervals $S_{q}$ for large $q$. We now seek to show this for the function $V(t)$.

USE OF LEMMA 12.

$$
\frac{3}{4} v\left(t_{q}\right) \leqslant v(t) \leqslant \frac{5}{4} v\left(t_{q}\right) .
$$

We make use of the fact that $v\left(t_{q}\right)$ tends to infinity and (a) so that we also have

$$
J \cdot=v^{2}+R V \cdot \geqslant v^{2}-\mu_{q} / r-a_{10}>v^{2}-2 \mu v / c>v^{2}\left(t_{q}\right) / 2 .
$$

So

$$
J \cdot>v^{2}\left(t_{q}\right) / 2 \text {. }
$$

LEMMA 13. The length of $S_{q}<a_{12} \delta\left(t_{q}\right) / v\left(t_{q}\right)$. 
Proof. Let

$$
J^{*}(t)=\left(v^{2}\left(t_{q}\right) / 4\right)\left(t-t_{q}\right)^{2}-\left(\delta\left(t_{q}\right) v\left(t_{q}\right)\right)\left(t-t_{q}\right)+\delta^{2}\left(t_{q}\right) / 2,
$$

defined for $t \in S_{q} . J\left(t_{q}\right)=J^{*}\left(t_{q}\right)$, since $r\left(t_{q}\right)=\delta\left(t_{q}\right) . J^{*} \cdot\left(t_{q}\right)<J \cdot\left(t_{q}\right)$ since

$$
J^{*}\left(t_{q}\right)=-\delta\left(t_{q}\right) v\left(t_{q}\right)<R\left(t_{q}\right) V\left(t_{q}\right)=J \cdot\left(t_{q}\right) .
$$

$J^{* \cdot \cdot}(t)<J \cdot \cdot(t)$ for $t \in S_{q}$ by definition of $J^{*}$ and (b). The preceding inequalities imply $J^{*}(t)<J(t), t \in S_{q}$. However, suppose length of $S_{q} \geqslant$ $5 \delta\left(t_{q}\right) / v\left(t_{q}\right)$. This would mean that there is a point of $S_{q}, T_{q}^{\prime \prime}$, with $t_{q}^{\prime \prime}-t_{q}=$ $5 \delta\left(t_{q}\right) / v\left(t_{q}\right)$ such that

$$
J^{*}\left(t_{q}^{\prime \prime}\right)=25 \delta^{2}\left(t_{q}\right) / 4-5 \delta^{2}\left(t_{q}\right)+\delta^{2}\left(t_{q}\right) / 2>\delta^{2}\left(t_{q}\right) / 2>J\left(t_{q}^{\prime \prime}\right)
$$

since

$$
J\left(t_{q}^{\prime \prime}\right)=r^{2}\left(t_{q}^{\prime \prime}\right) / 2 \leqslant \delta^{2}\left(t_{q}^{\prime \prime}\right) / 2<\delta^{2}\left(t_{q}\right) / 2 .
$$

This is a contradiction. Therefore length of $S_{q}<5 \delta\left(t_{q}\right) / v\left(t_{q}\right)$.

Corollary. For $t \in S_{q},\left|V(t)-V\left(t_{q}^{\prime}\right)\right|<a_{13} \delta\left(t_{q}\right) v\left(t_{q}^{\prime}\right)$.

Proof.

$$
\left|V(t)-V\left(t_{q}^{\prime}\right)\right| \leqslant \int_{t}^{t_{q}^{\prime}}|V \cdot(s)| d s
$$

and

$$
|V \cdot(s)| \leqslant \mu_{q} / r^{2}(s)+a_{10} / r(s)<2 \mu^{*} / r^{2}(s)
$$

where $\mu^{*}=$ supremum over $q$ of $\mu_{q}$. Also from Lemma 10(2), we have $r v \geqslant|R \times V| \geqslant c$ so $|V \cdot|<2 \mu^{*} v^{2} / c^{2}$.

$$
\begin{aligned}
\left|V(t)-V\left(t_{q}^{\prime}\right)\right| & <c^{-2} \int_{t}^{t_{q}^{\prime}} 2 \mu^{*} v^{2}(s) d s \\
& \left.<\frac{50}{9} \mu^{*} v^{2}\left(t_{q}^{\prime}\right) \text { (length of } S_{q}\right) / c^{2} \\
& \leqslant \frac{50}{9} \mu^{*} a_{12} v^{2}\left(t_{q}^{\prime}\right) \delta\left(t_{q}\right) /\left(c^{2} v\left(t_{q}\right)\right) \\
& <\frac{250}{36} \mu^{*} a_{12} \delta\left(t_{q}\right) v\left(t_{q}^{\prime}\right) / c^{2},
\end{aligned}
$$

where we use condition (a) to get $v(s)<5 v\left(t_{q}\right) / 4<(5 / 4)(4 / 3) v\left(t_{q}^{\prime}\right)$.

REMARK. The preceding corollary is a crucial step in showing that the solution which we are now considering is not a noncollision singularity. The corollary shows that $V$ is asymptotically constant with respect to its absolute value at $t_{q}^{\prime}$. We show with little additional work that $C_{\dot{s} p^{\prime}}^{\prime}, C_{p p^{\prime}}^{\cdot}$ are also asymptotically constant with respect to $v\left(t_{q}^{\prime}\right)$ but this kind of estimate is worthless unless $V\left(t_{q}^{\prime}\right),\left|C_{\dot{s}^{\prime}}\right|$ and $\left|C_{p^{\prime}}\right|$ are mutually comparable.

Notation. Let $W_{q}=W_{k_{q}}$ and $W_{q}^{\prime}=W_{k_{q}+1}$. Let $V_{1}=C_{i}, V_{2}=C_{\dot{2}}, V_{3}=$ $C_{3}, V_{12}=C_{12}, V_{13}=C_{i 3}, V_{23}=C_{23}$, and lower case $v$ 's denote absolute 
value. Let $V^{\prime}=V_{p^{\prime}}-V_{s}$ and $v^{\prime}=\left|V^{\prime}\right|$, defined for $t \in W_{q} \cup S_{q} \cup W_{q}^{\prime}$.

Let $\varepsilon_{0 q}$ be a positive sequence with limit 0 such that for $t \in S_{q}, \mid V(t)-$ $V\left(t_{q}^{\prime}\right) \mid<\varepsilon_{0 q} v\left(t_{q}^{\prime}\right)$. This inequality is a restatement of the corollary of Lemma 13.

LEMMA 14. We can find a sequence, $\varepsilon_{1 q}$, with limit equal to zero such that $\tau_{q} \leqslant t \leqslant \tau_{q}^{\prime}$ implies

$$
\left|V_{i j}(t)-V_{i j}\left(t_{q}^{\prime}\right)\right| \leqslant \varepsilon_{1 q} v\left(t_{q}^{\prime}\right), \quad 1<i<j<3 .
$$

REMARK. The proof of this lemma consists of nothing more than using the corollary to Lemma 13 and the known good behavior of $V_{1}, V_{2}, V_{3}$, on $W_{k}$.

Proof. We have the algebraic identities $V_{s}-V_{p}=V, M_{s} V_{s}+M_{p} V_{p}=$ - $M_{p^{\prime}} V_{p^{\prime}}$. We can write these equations in matrix forms, $A\left(V_{s}, V_{p}\right)=$ $\left(V, V_{p^{\prime}}\right)$ where $A$ is a $6 \times 6$ matrix. The matrix $A$ depends on the cluster masses and therefore depends on $q$, but as we have seen before, $A$ has only finitely many possible values. All of these possible matrices are invertible. Let $a_{14}=$ maximum over all possibilities of norm $\left(A^{-1}\right)$. Then if we write $\left(V, V_{p^{\prime}}\right)$ $=\left(V\left(t_{q}^{\prime}\right), V_{p^{\prime}}\left(t_{q}^{\prime}\right)\right)+W^{*}$ where $W^{*}$ is a map of $\left(\tau_{q}, \tau_{q}^{\prime}\right)$ into $R^{6}$ satisfying the preceding equation, then $\left|V_{s}(t)-V_{s}\left(t_{q}^{\prime}\right)\right|<a_{14}\left|W^{*}(t)\right|$ and $\left|V_{p}(t)-V_{p}\left(t_{q}^{\prime}\right)\right|<$ $a_{14}\left|W^{*}(t)\right|$. Let $W^{*}=\left(W_{1}^{*}, W_{2}^{*}\right)$ where $W_{1}^{*}$ is the first three components of $W^{*}$. By the definition of $\varepsilon_{0 q}$ for $t \in S_{q},\left|W_{1}^{*}(t)\right|<\varepsilon_{0 q} v\left(t_{q}^{\prime}\right)$. Since $G_{s}$ and $G_{p}$ are distinct clusters on $W_{q}$ and $W_{q}^{\prime}$, by Lemma 3 the function $v(t)$ is well behaved on $W_{q} \cup W_{q}^{\prime} ;\left|W_{1}^{*}(t)\right| \leqslant 2 a_{3} \delta\left(\tau_{q}\right)+\varepsilon_{0 q} v\left(t_{q}^{\prime}\right)$. The same is true of $V_{p^{\prime}}$ since $G_{p^{\prime}}$ is one of the three clusters on $W_{q} \cup W_{q}^{\prime}$ and equals $H^{\prime}$ on $S_{q}$. Therefore for $\tau_{q} \leqslant t \leqslant \tau_{q}^{\prime}$,

$$
\left|W_{2}^{*}(t)\right| \leqslant a_{3} \delta\left(\tau_{q}\right)+a_{4}\left(t_{q}^{\prime}-t_{q}\right)+a_{3} \delta\left(t_{q}^{\prime}\right) \leqslant 3 a_{3} \delta\left(\tau_{q}\right)<1 .
$$

Since $v\left(t_{q}^{\prime}\right)$ tends to infinity, we can certainly find a sequence, $\varepsilon_{q}^{\prime}$ with limit 0 such that for $\tau_{q}<t \leqslant \tau_{q}^{\prime},\left|W^{*}(t)\right|<\left(1 / a_{14}\right) \varepsilon_{q}^{\prime} v\left(t_{q}^{\prime}\right)$. A possible choice of $\varepsilon_{q}^{\prime}$ is $2 a_{14} \varepsilon_{0 q}$. We have just shown that $V_{p^{\prime}}$ is much better behaved than either velocity $V_{s}$ or $V_{p}$ so by setting $\varepsilon_{1 q}=2 \varepsilon_{q}^{\prime}$ we can satisfy the statement of this lemma.

LEMMA 15. There exists a positive number, $a_{15}$, such that $v^{\prime}\left(\tau_{q}\right)>(1+$ $\left.a_{15}\right) v\left(\tau_{q}\right)$.

Proof.

$$
\begin{aligned}
V^{\prime} & =\left(1 / M_{p^{\prime}}\right)\left(-M_{s} V_{s}-M_{p} V_{p}\right)-V_{s} \\
& =-\left(\left(1+M_{s} / M_{p^{\prime}}\right) V+\left(1+M_{s} / M_{p^{\prime}}+M_{p} / M_{p^{\prime}}\right) V_{p}\right) \\
& =-\left(1+\alpha_{1}\right) V-\left(1+\beta_{1}\right) V_{p} .
\end{aligned}
$$

The preceding are algebraic identities valid on $W_{q} \cup S_{q} \cup W_{q}^{\prime}, \alpha_{1}$ and $\beta_{1}$ depend on $M_{1}, M_{2}, M_{3}$, the masses of the three clusters, and therefore depend 
on $q$, but since there are only finitely many possibilities for $\alpha_{1}$ and $\beta_{1}$ and they are all positive, then $\alpha_{1}$ and $\beta_{1}$ are bounded away from 0 and infinity.

Let $\alpha^{*}=$ smallest possible value of $\alpha_{1}>m_{0} / M^{*}$. Then

$$
\begin{aligned}
v^{\prime 2} & =\left(1+\alpha_{1}\right)^{2} v^{2}+2\left(1+\alpha_{1}\right)\left(1+\beta_{1}\right) V V_{p}+\left(1+\beta_{1}\right)^{2} v_{p}^{2} \\
& \geqslant\left(1+2 \alpha^{*}\right) v^{2}+\beta_{2} V V_{p}+v_{p}^{2}
\end{aligned}
$$

where $\beta_{2}=2\left(1+\alpha_{1}\right)\left(1+\beta_{1}\right)$. If $a_{15}=\sqrt{1+\alpha^{*}}-1$ then the lemma would be proved if we could show $\alpha^{*} v^{2}\left(\tau_{q}\right)+\beta_{2} V V_{p}+v_{p}^{2}>0$. The only term which is not automatically positive is $V V_{p}$. We will show that $V\left(\tau_{q}\right)=$ $\gamma_{1} C_{p}\left(\tau_{q}\right)+$ a small error term. If we then recall that one of the two clusters of $S_{k_{q}-1}$ must be $G_{p}$, and use Lemma 7 to throw away most of the term $V V_{p}$, as it will be positive, what is left will be the error term times $-\beta_{2} v\left(\tau_{q}\right)$ which we can overcome with $v^{2}$ and $v_{p}^{2}$.

Recall $V=C_{s p}=C_{s}-C_{p}$. Then

$$
C_{s p}\left(t_{q}\right)=C_{s p}\left(\tau_{q}\right)+\int_{W_{q}} V(\nu) d(\nu) .
$$

Let $V^{*}=V(t)-V\left(\tau_{q}\right)$. From Lemma $3\left|V^{*}\right|=v^{*}<2 a_{3} \delta\left(\tau_{q}\right)$. Then

$$
C_{s p}\left(\tau_{q}\right)=C_{s p}\left(t_{q}\right)-\int_{\tau_{q}}^{t_{q}} V^{*}(\nu) d \nu-V\left(\tau_{q}\right)\left(t_{q}-\tau_{q}\right)
$$

Let $\theta_{q}=C_{s p}\left(t_{q}\right)-\int_{\tau_{q}}^{t_{q}} V^{*}(\nu) d \nu$. We estimate

$$
\left|\theta_{q}\right|<\left|C_{s p}\left(t_{q}\right)+\left(t_{q}-\tau_{q}\right)\left(2 a_{3} \delta\left(\tau_{q}\right)\right)\right|<2 \delta\left(\tau_{q}\right)
$$

since $\left(t_{q}-\tau_{q}\right)<-\tau_{q}=\delta^{3}\left(\tau_{q}\right)$ and $\left|C_{s p}\left(t_{q}\right)\right|=\delta\left(t_{q}\right) \leqslant \delta\left(\tau_{q}\right)$. Then $C_{s p}\left(\tau_{q}\right)$ $=-V\left(\tau_{q}\right)\left(t_{q}-\tau_{q}\right)+\theta_{q}$. This is very nearly the expression which we need for $C_{p}\left(\tau_{q}\right)$, but $C_{s p}\left(\tau_{q}\right)$ is - const. $C_{p}\left(\tau_{q}\right)+$ small error term. Let

$$
D_{q}^{*}=\left(1 /\left(M_{s}+M_{p^{\prime}}\right)\right)\left(M_{s} C_{s}\left(\tau_{q}\right)+M_{p^{\prime}} C_{p^{\prime}}\left(\tau_{q}\right)\right) \text {. }
$$

The quantity $D_{q}^{*}$ is the center of mass of one of the clusters $H_{k_{q}-1}, H_{k_{q}-1}^{\prime}$ which is the union of $G_{s}$ and $G_{p^{\prime}}$ at time $\tau_{q}$. What we will use is $C_{s}\left(\tau_{q}\right)=D_{q}^{*}$ $+\theta_{q}^{\prime}$ where $\left|\theta_{q}^{\prime}\right|<c_{s p^{\prime}}\left(\tau_{q}\right)=\delta\left(\tau_{q}\right)$; this is possible because $D_{q}^{*}$ is a convex combination of $C_{s}\left(\tau_{q}\right)$ and $C_{p^{\prime}}\left(\tau_{q}\right)$. Then

$$
C_{s p}\left(\tau_{q}\right)=D_{q}^{*}-C_{p}\left(\tau_{q}\right)+\theta_{q}^{\prime}=-\left(1+M_{p} /\left(M_{s}+M_{p^{\prime}}\right)\right) C_{p}\left(\tau_{q}\right)+\theta_{q}^{\prime} .
$$

Let $\beta_{3}=\left(1 /\left(1+M_{p} /\left(M_{s}+M_{p^{\prime}}\right)\right)\right)$. Then $\beta_{3}$ is positive and

$$
C_{p}\left(\tau_{q}\right)=-\beta_{3} C_{s p}\left(\tau_{q}\right)-\beta_{3} \theta_{q}^{\prime}=\beta_{3}\left(V\left(\tau_{q}\right)\left(t_{q}-\tau_{q}\right)-\theta_{q}\right)-\beta_{3} \theta_{q}^{\prime} .
$$

Since $\beta_{3}<1$,

$$
C_{p}\left(\tau_{q}\right)=\beta_{3} V\left(\tau_{q}\right)\left(t_{q}-\tau_{q}\right)+\theta_{q}^{\prime \prime} \text { and }\left|\theta_{q}^{\prime \prime}\right|<a_{16} \delta\left(\tau_{q}\right)
$$

Divide both sides of this equation to express

$$
V\left(\tau_{q}\right)=\left(1 / \beta_{3}\right) C_{p}\left(\tau_{q}\right)\left(t_{q}-\tau_{q}\right)+V_{q}
$$


where

$$
V_{q}=-\left(1 /\left(\beta_{3}\left(t_{q}-\tau_{q}\right)\right)\right)\left(\theta_{q}^{\prime \prime}\right)
$$

and since $\beta_{3}>1 /\left(1+\left(M^{*} / m_{0}\right)\right)$, we have

$$
\left|V_{q}\right|=v_{q}<a_{16}^{\prime} \delta\left(\tau_{q}\right) /\left(t_{q}-\tau_{q}\right) \text {. }
$$

We need only one more fact about $V\left(\tau_{q}\right)$ to complete the proof. That is that

$$
\begin{aligned}
v\left(\tau_{q}\right) & >\sup _{\nu \in W_{q}} v(\nu)-2 a_{3} \delta\left(\tau_{q}\right) \\
& \geqslant\left|C_{s p}\left(\tau_{q}\right)-C_{s p}\left(t_{q}\right)\right| /\left(t_{q}-\tau_{q}\right)-2 a_{3} \delta\left(\tau_{q}\right) \\
& >a_{1} /\left(3\left(t_{q}-\tau_{q}\right)\right) .
\end{aligned}
$$

This is true because $\left|C_{s p}\left(\tau_{q}\right)\right|>a_{1} / 2$ and $C_{s p}\left(t_{q}\right)=\delta\left(t_{q}\right)$.

Recall we wish to show

$$
\alpha^{*} v^{2}\left(\tau_{q}\right)+\beta_{2} V\left(\tau_{q}\right) V_{p}\left(\tau_{q}\right)+v_{p}^{2}\left(\tau_{q}\right)>0 .
$$

Since $V\left(\tau_{q}\right)=\left(1 / \beta_{3}\right) C_{p}\left(\tau_{q}\right) /\left(t_{q}-\tau_{q}\right)+V_{q}$,

$$
\begin{aligned}
\beta_{2} V\left(\tau_{q}\right) V_{p}\left(\tau_{q}\right) & =\beta_{2} V_{p}\left(\tau_{q}\right) V_{q}+\left(\beta_{2} / \beta_{3}\right) V_{p}\left(\tau_{q}\right) C_{p}\left(\tau_{q}\right) /\left(t_{q}-\tau_{q}\right) \\
& \geqslant-\beta_{2}^{*} v_{p}\left(\tau_{q}\right) v_{q}
\end{aligned}
$$

where $\beta_{2}^{*}$ is the greatest possible value of $\beta_{2}$. To do this we have used Lemma 7 which tells us $V_{p}\left(\tau_{q}\right) C_{p}\left(\tau_{q}\right)$ tends to positive infinity so it is certainly positive for large $q$. Finally the polynomial in $v_{p}\left(\tau_{q}\right), v_{p}^{2}\left(\tau_{q}\right)-\beta_{2}^{*} v_{q} v_{p}\left(\tau_{q}\right)$, is bounded below by $-\beta_{2}^{* 2} v_{q}^{2} / 4>-a_{17} \delta^{2}\left(\tau_{q}\right) /\left(t_{q}-\tau_{q}\right)^{2}$. This bound is independent of $v_{p}$. We have just shown that $v\left(\tau_{q}\right)>a_{1} /\left(3\left(t_{q}-\tau_{q}\right)\right)$ so if we take $q$ large enough that $\alpha^{*} a_{1}^{2} / 9>a_{17} \delta^{2}\left(\tau_{q}\right)$, we then have $\alpha^{*} v^{2}\left(\tau_{q}\right)+\beta_{2} V\left(\tau_{q}\right) V_{p}\left(\tau_{q}\right)+$ $v_{p}^{2}\left(\tau_{q}\right)$ positive which proves the lemma.

COROLlaRY 1. $v_{s p}\left(t_{q}^{\prime}\right)>\left(1+a_{15} / 2\right) v\left(t_{q}^{\prime}\right)$.

Proof.

$$
\begin{aligned}
v_{s p^{\prime}}\left(t_{q}^{\prime}\right) & >v_{s p^{\prime}}\left(\tau_{q}\right)-\varepsilon_{1 q} v\left(t_{q}^{\prime}\right) \\
& >\left(1+a_{15}\right)\left(v\left(t_{q}^{\prime}\right)-\varepsilon_{0 q} v\left(t_{q}^{\prime}\right)-\varepsilon_{1 q} v\left(t_{q}^{\prime}\right)\right)-\varepsilon_{1 q} v\left(t_{q}^{\prime}\right) \\
& >\left(1+a_{15} / 2\right) v\left(t_{q}^{\prime}\right) .
\end{aligned}
$$

COROllary 2. $v_{p p^{\prime}}\left(t_{q}^{\prime}\right)>a_{15} v\left(t_{q}^{\prime}\right) / 2$.

Proof. Corollary 2 follows from Corollary 1 and the triangle inequality.

COROllary 3. We can find a sequence $\varepsilon_{2 q}$ with limit 0 such that $\mid V_{i j}(t)-$ $V_{i j}\left(t_{q}^{\prime}\right) \mid<\varepsilon_{2 q} v_{i j}\left(t_{q}^{\prime}\right)$ for $\tau_{q} \leqslant t \leqslant \tau_{q}^{\prime}$ and $i, j=1,2,3$.

Proof. Let $\varepsilon_{2 q}=2 \varepsilon_{1 q} / a_{15}$. The corollary then follows from Lemma 14 and Corollaries 1 and 2 of Lemma 15. 
6. In $\S 5$, Theorem 1 was assumed to be false. From this, it was deduced that the cluster velocities, $V_{i}, i=1,2,3$, undergo little change on the interval $S_{q}$. Lemmas 17 and 18 show that the cluster velocities must undergo radical changes on each interval, $S_{k}$, in order for the clusters to ever again reform. Since the clusters must regroup in order for the solution to be a noncollision singularity, the contradiction is apparent.

LemMa 16. Suppose $F(t)$ is a $C_{1}$ map of a real interval into $R^{n}$. Let $t_{1}$ and $t_{2}$ be the endpoints of this interval, not necessarily numbered in left, right order. Moreover, suppose $\left|F\left(t_{1}\right)\right|=\delta$ and $\left|F \cdot(t)-F \cdot\left(t_{2}\right)\right|<\varepsilon\left|F \cdot\left(t_{2}\right)\right|$. Finally suppose $\delta<\varepsilon\left|F \cdot\left(t_{2}\right)\right|\left|t_{2}-t_{1}\right|$. Then $F\left(t_{2}\right)=F \cdot\left(t_{2}\right)\left(t_{2}-t_{1}\right)+\eta$ where $|\eta| \leqslant$ $2 \varepsilon\left|F \cdot\left(t_{2}\right)\left(t_{2}-t_{1}\right)\right|$.

REMARKs. This lemma may seem entirely trivial and so it is, but we include it because it will streamline the last part of the proof of Theorem 1 .

Proof.

$$
\begin{aligned}
F\left(t_{2}\right) & =F\left(t_{1}\right)+\int_{t_{1}}^{t_{2}} F \cdot(\nu) d \nu \\
& =F\left(t_{1}\right)+F \cdot\left(t_{2}\right)\left(t_{2}-t_{1}\right)+\int_{t_{1}}^{t_{2}}\left(F \cdot(\nu)-F \cdot\left(t_{2}\right)\right) d \nu \\
& =F \cdot\left(t_{2}\right)\left(t_{2}-t_{1}\right)+\eta
\end{aligned}
$$

where $\eta=F\left(t_{1}\right)+\int_{t_{1}}^{t_{2}}\left(F \cdot(\nu)-F \cdot\left(t_{2}\right)\right) d \nu$. Therefore

$$
|\eta| \leqslant\left|F\left(t_{1}\right)\right|+\left|\int_{t_{2}}^{t_{1}}\left(F \cdot(\nu)-F \cdot\left(t_{2}\right)\right) d \nu\right|<\delta+\varepsilon\left|F \cdot\left(t_{2}\right)\left(t_{2}-t_{1}\right)\right|
$$

which by hypothesis is less than or equal to $2 \varepsilon\left|F^{\cdot}\left(t_{2}\right)\left(t_{2}-t_{1}\right)\right|$.

Notation. Let $K$ be the unique integer, 1, 2, 3, such that $H_{k_{q}+1}=G_{K} \cup G_{p^{\prime}}$. Recall $H_{i}$ is the cluster on $S_{i}$ composed of two of the clusters of $W_{i}$. Note that Lemma 5 justifies the assertion that one of the clusters of $W_{q}^{\prime}$ which is included in $H_{k_{q}+1}$ is $G_{p^{\prime}} . K=s$ or $K=p$, also by Lemma 5. Let

$$
\varepsilon_{q}=\max \left(\varepsilon_{2 q}, \frac{\delta\left(\tau_{q}\right)}{v_{s p^{\prime}}\left(t_{q}^{\prime}\right)\left(t_{q}^{\prime}-\tau_{q}\right)}, \frac{\delta\left(t_{q}^{\prime}\right)}{v_{K p^{\prime}}\left(t_{q}^{\prime}\right)\left(\tau_{q}^{\prime}-t_{q}^{\prime}\right)}\right) .
$$

By the mean value theorem and Lemma 3,

$$
v_{K p^{\prime}}\left(t_{q}^{\prime}\right)\left(\tau_{q}^{\prime}-t_{q}^{\prime}\right)>\left|C_{K p^{\prime}}\left(t_{q}^{\prime}\right)-C_{K p^{\prime}}\left(\tau_{q}^{\prime}\right)\right|-2 a_{3} \delta\left(t_{q}^{\prime}\right)\left(\tau_{q}^{\prime}-t_{q}^{\prime}\right)>a_{1} / 3 .
$$

Similarly

$$
v_{s p^{\prime}}\left(t_{q}^{\prime}\right)\left(t_{q}^{\prime}-\tau_{q}\right)>\left(1-\varepsilon_{2 q}\right)\left|C_{s p^{\prime}}\left(t_{q}^{\prime}\right)-C_{s p^{\prime}}\left(\tau_{q}\right)\right|>a_{1} / 3
$$

Here instead of Lemma 3 we must use Corollary 3 of Lemma 15. The above two inequalities assure that $\varepsilon_{q}$ tends to zero. Further notice that the hypothe- 
ses of Lemma 16 are satisfied by $\delta=\delta\left(\tau_{q}\right), \varepsilon=\varepsilon_{q}, t_{1}=\tau_{q}, t_{2}=t_{q}^{\prime}, F(t)=$ $C_{s p^{\prime}}(t)$, and by $\delta=\delta\left(\tau_{q}^{\prime}\right), \varepsilon=\varepsilon_{q}, t_{1}=\tau_{q}^{\prime}, t_{2}=t_{q}^{\prime}, F(t)=C_{K p^{\prime}}(t)$.

\section{LEMMA 17.}

$$
\lim _{q \rightarrow \infty} \frac{C_{s p^{\prime}}\left(t_{q}^{\prime}\right) V_{s p^{\prime}}\left(t_{q}^{\prime}\right)}{c_{s p^{\prime}}\left(t_{q}^{\prime}\right) v_{s p^{\prime}}\left(t_{q}^{\prime}\right)}=1 \text { and } \lim _{q \rightarrow \infty} \frac{C_{K p^{\prime}}\left(t_{q}^{\prime}\right) V_{K p^{\prime}}\left(t_{q}^{\prime}\right)}{c_{K p^{\prime}}\left(t_{q}^{\prime}\right) v_{K p^{\prime}}\left(t_{q}^{\prime}\right)}=-1 \text {. }
$$

REMARKS. This lemma rules out $K=s$, and is used in the proof of Lemma 18.

Proof. Apply Lemma 16 to the function $C_{s p^{\prime}}(t)$ on the interval $\left(\tau_{q}, t_{q}^{\prime}\right)$ with $\delta=\delta\left(\tau_{q}\right)$ and $\varepsilon=\varepsilon_{q}$. Then use Lemma 16 to write

$$
C_{s p^{\prime}}\left(t_{q}^{\prime}\right)=V_{s p^{\prime}}\left(t_{q}^{\prime}\right)\left(t_{q}^{\prime}-\tau_{q}\right)+\eta_{q}
$$

where

$$
\left|\eta_{q}\right|<2 \varepsilon_{q}\left|V_{s p^{\prime}}\left(t_{q}^{\prime}\right)\right|\left(t_{q}^{\prime}-\tau_{q}\right)
$$

Now

$$
\frac{V_{s p^{\prime}}\left(t_{q}^{\prime}\right) C_{s p^{\prime}}\left(t_{q}^{\prime}\right)}{\left\{v_{s p^{\prime}}\left(t_{q}^{\prime}\right) c_{s p^{\prime}}\left(t_{q}^{\prime}\right)\right\}}=\frac{\left(v_{s p^{\prime}}^{2}\left(t_{q}^{\prime}\right)\left(t_{q}^{\prime}-\tau_{q}\right)+V_{s p^{\prime}}\left(t_{q}^{\prime}\right) \eta_{q}\right)}{\left\{v_{s p^{\prime}}\left(t_{q}^{\prime}\right)\left|V_{s p^{\prime}}\left(t_{q}^{\prime}\right)\left(t_{q}^{\prime}-\tau_{q}\right)+\eta_{q}\right|\right\}}>\left(1-2 \varepsilon_{q}\right) /\left(1+2 \varepsilon_{q}\right)
$$

which proves part 1 of the lemma since $\varepsilon_{q}$ tends to 0 , and $V_{s p^{\prime}} C_{s p^{\prime}}<v_{s p^{\prime}} c_{s p^{\prime}}$. Similarly, apply Lemma 16 to the interval $\left(t_{q}^{\prime}, \tau_{q}^{\prime}\right)$ with $t_{1}=\tau_{q}^{\prime}, t_{2}=t_{q}^{\prime}$. Let $F(t)=C_{K p^{\prime}}(t), \delta=\delta\left(\tau_{q}^{\prime}\right)$ and $\varepsilon=\varepsilon_{q}$. Then use Lemma 16 to express

$$
C_{K p^{\prime}}\left(t_{q}^{\prime}\right)=V_{K p^{\prime}}\left(t_{q}^{\prime}\right)\left(t_{q}^{\prime}-\tau_{q}^{\prime}\right)+\eta_{q}^{\prime}
$$

with

$$
\left|\eta_{q}^{\prime}\right| \leqslant 2 \varepsilon_{q}\left|V_{K p^{\prime}}\left(t_{q}^{\prime}\right)\right|\left(\tau_{q}^{\prime}-t_{q}^{\prime}\right)
$$

Then

$$
\begin{aligned}
\frac{V_{K p^{\prime}}\left(t_{q}^{\prime}\right) C_{K p^{\prime}}\left(t_{q}^{\prime}\right)}{v_{K p^{\prime}}\left(t_{q}^{\prime}\right) c_{K p^{\prime}}\left(t_{q}^{\prime}\right)} & =\frac{v_{K p^{\prime}}^{2}\left(t_{q}^{\prime}\right)\left(t_{q}^{\prime}-\tau_{q}^{\prime}\right)+V_{K p^{\prime}}\left(t_{q}^{\prime}\right) \eta_{q}^{\prime}}{v_{K p^{\prime}}\left(t_{q}^{\prime}\right)\left|V_{K p^{\prime}}\left(t_{q}^{\prime}\right)\left(t_{q}^{\prime}-\tau_{q}^{\prime}\right)+\eta_{q}^{\prime}\right|} \\
& \leqslant \frac{v_{K p^{\prime}}^{2}\left(t_{q}^{\prime}\right)\left(t_{q}^{\prime}-\tau_{q}^{\prime}\right)+v_{K p^{\prime}}\left(t_{q}^{\prime}\right)\left|\eta_{q}^{\prime}\right|}{v_{K p^{\prime}}^{2}\left(t_{q}^{\prime}\right)\left(\tau_{q}^{\prime}-t_{q}^{\prime}\right)+v_{K p^{\prime}}\left(t_{q}^{\prime}\right)\left|\eta_{q}^{\prime}\right|} \leqslant \frac{-1+2 \varepsilon_{q}}{1+2 \varepsilon_{q}} .
\end{aligned}
$$

This proves the second part of the lemma since

$$
V_{K p^{\prime}}\left(t_{q}^{\prime}\right) C_{K p^{\prime}}\left(t_{q}^{\prime}\right) /\left(v_{K p^{\prime}}\left(t_{q}^{\prime}\right) c_{K p^{\prime}}\left(t_{q}^{\prime}\right)\right)>-1 .
$$

FINAL STEP IN PROOF OF THEOREM 1. By the remark preceding the proof of Lemma 17, we know that Lemma 17 implies $K=p$. We assume this and then show that for large $q, V_{p p^{\prime}}\left(t_{q}^{\prime}\right) C_{p p^{\prime}}\left(t_{q}^{\prime}\right)$ is positive. To do this we use part 1 of Lemma 17 and the corollary to Lemma 14. Naturally, the statement that $V_{p p^{\prime}}\left(t_{q}^{\prime}\right) C_{p p^{\prime}}\left(t_{q}^{\prime}\right)>0$ for all large $q$ contradicts Lemma 17 . 
LEMMA 18. $V_{p p^{\prime}}\left(t_{q}^{\prime}\right) C_{p p^{\prime}}\left(t_{q}^{\prime}\right)>0$ for all large $q$.

Proof. By definition of $V, V_{p p^{\prime}}=V_{s p^{\prime}}-V$. Let

$$
\varphi=C_{p p^{\prime}}\left(t_{q}^{\prime}\right)-C_{s p^{\prime}}\left(t_{q}^{\prime}\right)=-C_{s p}\left(t_{q}^{\prime}\right)
$$

Therefore $|\varphi|=\delta\left(t_{q}^{\prime}\right)$. Now we have

$$
C_{p p^{\prime}}\left(t_{q}^{\prime}\right) V_{p p^{\prime}}\left(t_{q}^{\prime}\right)=V_{s p^{\prime}}\left(t_{q}^{\prime}\right) C_{s p^{\prime}}\left(t_{q}^{\prime}\right)-V\left(t_{q}^{\prime}\right) C_{s p^{\prime}}\left(t_{q}^{\prime}\right)-V\left(t_{q}^{\prime}\right) \varphi+V_{s p^{\prime}}\left(t_{q}^{\prime}\right) \varphi .
$$

By part 1, Lemma 17, we can find a sequence, $\beta_{q}$, with limit 0 such that

$$
V_{s p^{\prime}}\left(t_{q}^{\prime}\right) C_{s p^{\prime}}\left(t_{q}^{\prime}\right)>\left(1-\beta_{q}\right) v_{s p^{\prime}}\left(t_{q}^{\prime}\right) c_{s p^{\prime}}\left(t_{q}^{\prime}\right) \text {. }
$$

Now write

$$
\begin{aligned}
& V_{s p^{\prime}}\left(t_{q}^{\prime}\right) C_{s p^{\prime}}\left(t_{q}^{\prime}\right)-V\left(t_{q}^{\prime}\right) C_{s p^{\prime}}\left(t_{q}^{\prime}\right)-V\left(t_{q}^{\prime}\right) \varphi+V_{s p^{\prime}}\left(t_{q}^{\prime}\right) \varphi \\
& >\left(1-\beta_{q}\right) v_{s p^{\prime}}\left(t_{q}^{\prime}\right) c_{s p^{\prime}}\left(t_{q}^{\prime}\right)-v_{s p^{\prime}}\left(t_{q}^{\prime}\right) \delta\left(t_{q}^{\prime}\right)-v\left(t_{q}^{\prime}\right) c_{s p^{\prime}}\left(t_{q}^{\prime}\right)-\delta\left(t_{q}^{\prime}\right) v\left(t_{q}^{\prime}\right) \\
& \quad=\left(1-\beta_{q}-\delta\left(t_{q}^{\prime}\right) / c_{s p^{\prime}}\left(t_{q}^{\prime}\right)\right) v_{s p^{\prime}}\left(t_{q}^{\prime}\right) c_{s p^{\prime}}\left(t_{q}^{\prime}\right)-\left(1+\delta\left(t_{q}^{\prime}\right) / c_{s p^{\prime}}\left(t_{q}^{\prime}\right)\right) v\left(t_{q}^{\prime}\right) c_{s p^{\prime}}\left(t_{q}^{\prime}\right) .
\end{aligned}
$$

Observe that since $c_{s p^{\prime}}\left(t_{q}^{\prime}\right)>a_{1} / 2-2 \delta\left(t_{q}^{\prime}\right)>a_{1} / 3$, we certainly have $\delta\left(t_{q}^{\prime}\right) / c_{s p^{\prime}}\left(t_{q}^{\prime}\right)$ tends to 0 . Therefore we let $\beta_{q}^{\prime}=\delta\left(t_{q}^{\prime}\right) / c_{s p}\left(t_{q}^{\prime}\right)$ and $\beta_{q}^{\prime \prime}=\beta_{q}+$ $\beta_{q}^{\prime}$. We can write

$$
V_{p p^{\prime}}\left(t_{q}^{\prime}\right) C_{p p^{\prime}}\left(t_{q}^{\prime}\right)>\left(1-\beta_{q}^{\prime \prime}\right) v_{s p^{\prime}}\left(t_{q}^{\prime}\right) c_{s p^{\prime}}\left(t_{q}^{\prime}\right)-\left(1+\beta_{q}^{\prime}\right) v\left(t_{q}^{\prime}\right) c_{s p^{\prime}}\left(t_{q}^{\prime}\right) .
$$

By Corollary 1 to Lemma $15, v_{s p^{\prime}}\left(t_{q}^{\prime}\right)>\left(1+a_{15} / 2\right) v\left(t_{q}^{\prime}\right)$ so

$$
\begin{aligned}
V_{p p^{\prime}}\left(t_{q}^{\prime}\right) C_{p p^{\prime}}\left(t_{q}^{\prime}\right) & \geqslant\left(1-\beta_{q}^{\prime \prime}\right)\left(1+a_{15} / 2\right) v\left(t_{q}^{\prime}\right)\left(c_{s p^{\prime}}\left(t_{q}^{\prime}\right)\right)-\left(1+\beta_{q}^{\prime}\right) v\left(t_{q}^{\prime}\right) c_{s p^{\prime}}\left(t_{q}^{\prime}\right) \\
& \geqslant\left(\left(1-\beta_{q}^{\prime \prime}\right)\left(1+a_{15} / 2\right)-\left(1+\beta_{q}^{\prime}\right)\right) v\left(t_{q}^{\prime}\right) c_{s p^{\prime}}\left(t_{q}^{\prime}\right)
\end{aligned}
$$

which is positive in view of the fact that $\beta_{q}^{\prime}$ and $\beta_{q}^{\prime \prime}$ are tending to 0 .

\section{THEOREM 2}

Notation. For the remainder of the paper we will reserve the symbols $L$ and $L_{k}$ to designate lines. Moreover, for any point $X$ in $R^{n},|X-L|=\min \{\mid X-$ $Z|| Z \in L\}$. We also have for $X, Y$ in $R^{n},|X-L| \leqslant|X-Y|+|Y-L|$.

THEOREM 2. If $t=0$ is a noncollision singularity of the four-body problem then there exists a line $L$ through the origin such that $\lim _{t \rightarrow 0}\left|R_{i}(t)-L\right|=0$ for $i=1,2,3,4$.

REMARK. The proof of Theorem 2 will naturally use Theorem 1. It is worth noting that the only part of Theorem 1 which is critical to the proof of Theorem 2 is that the angular momentum of each cluster $H$ and $H^{\prime}$ is bounded independent of $t$.

Notation. We keep much of the notation of Theorem 1. The points $T_{1 k}$ and $T_{2 k}$ are the endpoints of $W_{k} ; G_{1}, G_{2}, G_{3}$ are the clusters on $W_{k}$ with $M_{i}$ the total mass of $G_{i}$ and $C_{i}$ the coordinate of the center of mass of $G_{i}$. Also define 
$C_{i j}$, $c_{i j}$ from $C_{i}$ as before. $H=G_{s} \cup G_{p}=$ one of the clusters on $S_{k}, 1 \leqslant s<$ $p<3 . H^{\prime}=G_{p^{\prime}}=$ other clusters on $S_{k}, p^{\prime}=6-s-p . D, D^{\prime}$ are the centers of mass of $H, H^{\prime}$, respectively. $Z, Z^{\prime}$ is the angular. momentum of $H$, $H^{\prime}$ with respect to $D, D^{\prime}$, respectively. We also keep the labeling convention that $G_{s} \cup G_{p^{\prime}}$ is one of the clusters $H, H^{\prime}$ on $S_{k-1}$. We therefore let $D_{-1}=\left(1 /\left(M_{s}+M_{p}\right)\right)\left(M_{s} C_{s}+M_{p^{\prime}} C_{p^{\prime}}\right)$ and $D_{-1}^{\prime}=-\left(M_{s}+M_{p^{\prime}}\right) D_{-1} / M_{p^{\prime}}$. $D_{-1}$ and $D_{-1}^{\prime}$ are the centers of mass of the clusters $H, H^{\prime}$ on $S_{k-1}$. The primes on $D$ do not necessarily agree with those on $H$ since we do not know the clustering arrangement on $W_{k-1} . R=C_{s p}, V=R ; r=|R|$, and $v=|V|$. Also keep $d(t)=\min \left\{r_{i j}(t) \mid 1 \leqslant i<j \leqslant 4\right\}$, and define $T_{k}$ as in Lemma 10 , part 1.

7. In this section we show under the assumption that the angular momentum of each cluster, $H_{k}$, with respect to its center of mass is bounded, that the cluster velocities, $C_{i}, i=1,2,3$, undergo changes which are bounded independent of $K$ as long as the clusters $H, H^{\prime}$ are larger than $1 /\left|C_{s p}\left(t_{2 k}\right)\right|$. The preceding bound on the size of clusters is crucial to showing the convergence of the particle positions to a fixed line.

LEMMA 19. If $T_{2 k} \leqslant t \leqslant T_{k}$ then $r(t)<-v(t) / 2$.

Proof. We shall use the identity $|A|^{2}|B|^{2}=(A B)^{2}+(A \times B)^{2}$ for points $A$ and $B$ in $R^{3}$. By hypothesis $r(t) v(t)>1$. By Theorem 1 and part 1 of Lemma 10, $R \times V$ tends to 0 as $t$ tends to 0 through the closed intervals [ $T_{2 k}, T_{k}$ ] so we can assume that $|R \times V|<\frac{1}{2}$. We then have $(R V)^{2}=r^{2} v^{2}-(R \times V)^{2}$ $\geqslant 3 r^{2} v^{2} / 4$ for $t$ as restricted by the hypothesis. Therefore since $r=R V / r$ and $r\left(T_{2 k}\right) \leqslant \delta \cdot\left(T_{2 k}\right)<0$, we have more than the claim of the corollary.

LEMMA 20. Let $S_{k}^{\prime}=\left\{t \in S_{k} \mid r(t) v(t)<1\right\}$. Then for sufficiently large $k, S_{k}^{\prime}$ is not empty.

Proof. Suppose $S_{k}^{\prime}$ empty. Then $T_{k}=T_{1 k+1}$ and by Lemma 10. part 1, the minimum distance is unique on $S_{k}$. Therefore the clusters $G_{1}, G_{2}, G_{3}$ on $W_{k}$ are also natural on $W_{k+1}$. Also by Lemma $5, c_{s p}\left(T_{2 k+1}\right) \geqslant a_{1} / 2$. Therefore

$$
v\left(T_{1 k+1}\right)=\left|C_{s p}\left(T_{1 k+1}\right)\right| \geqslant a_{1} /\left(3\left(T_{2 k+1}-T_{1 k+1}\right)\right)>a_{1} /\left(3 \delta^{3}\left(T_{1 k+1}\right)\right) .
$$

By the corollary to Theorem $1, r\left(T_{1 k+1}\right)<-a_{1} /\left(6 \delta^{3}\left(T_{1 k+1}\right)\right)$. On the other hand,

$$
r \cdot\left(T_{1 k+1}\right) \geqslant \delta \cdot\left(T_{1 k+1}\right)=-1 /\left(3 \delta^{2}\left(T_{1 k+1}\right)\right) .
$$

Both statements cannot hold since $\delta\left(T_{1 k+1}\right)$ tends to 0 , therefore $S_{k}^{\prime}$ cannot be empty for large $k$.

Corollary. $r\left(T_{k}\right) v\left(T_{k}\right) \leqslant 1$. 
Proof. This follows from the definition of $T_{k}, S_{k}^{\prime}$ not empty and continuity of $r$ on the closed interval [ $\left.T_{2 k}, T_{k}\right]$. Note we have not excluded the possibility that $T_{k}=T_{2 k}$.

LEMMA 21. If $T_{2 k}<t<T_{k}$, then $\left|V(t)-V\left(T_{2 k}\right)\right|<16 M^{* 2} / m_{0}$.

Proof. Let $r^{\prime}(t)=\min \left\{r_{i j}(t) \mid i \in G_{s}, j \in G_{p}\right\}$. We have

$$
\left|V(t)-V\left(T_{2 k}\right)\right|<\int_{T_{2 k}}^{t}\left(M^{* 2} / m_{0} r^{\prime 2}(s)\right) d s .
$$

Since $r>1 / v>1 /\left(a_{5} U^{1 / 2}\right)>d^{1 / 2} /\left(a_{5} M^{*}\right)$, we have $r^{2}>(r-d)^{2}>$ $r^{2} / 2$. We now have

$$
\left|V(t)-V\left(T_{2 k}\right)\right|<\int_{T_{2 k}}^{t}\left(2 M^{* 2} / m_{0} r^{2}(s)\right) d s .
$$

Assume Lemma 21 is false. Let

$$
\tau=\inf \left\{t \in\left[T_{2 k}, T_{k}\right]|| V(t)-V\left(T_{2 k}\right) \mid>16 M^{* 2} / m_{0}\right\}
$$

The supposition that Lemma 21 is false implies the set of which $\tau$ is the infimum cannot be empty. Also if Lemma 21 is false, we may assume $T_{2 k}$ does not belong to $S_{k}^{\prime}$, for if $T_{2 k} \in S_{k}^{\prime}$, then $T_{k}=T_{2 k}$, and Lemma 21 is automatically true. By continuity $\left|V(\tau)-V\left(T_{2 k}\right)\right|=16 M^{* 2} / m_{0}$. We further require $k$ large enough so that $v\left(T_{2 k}\right)>32 M^{* 2} / m_{0}$. We can do this because $v\left(T_{2 k}\right)>1 / r\left(T_{2 k}\right)=1 / \delta\left(T_{2 k}\right)$ tends to infinity. Here we use our observation that $T_{2 k}$ does not belong to $S_{k}^{\prime}$. We now have $v(t)>v(\tau) / 3$. We use these facts to approximate a solution of the inequality

$$
\left|V(\tau)-V\left(T_{2 k}\right)\right|<\int_{T_{2 k}}^{\tau}\left(2 M^{* 2} / m_{0} r^{2}(s)\right) d s .
$$

Multiply the right side of this inequality by $-2 r / v$ which is greater than 1 and write

$$
\begin{aligned}
\left|V(\tau)-V\left(T_{2 k}\right)\right| & \leqslant \frac{4 M^{* 2}}{m_{0}} \int_{T_{2 k}}^{\tau} \frac{-r(s) d s}{v(s) r^{2}(s)}<\frac{12 M^{* 2}}{m_{0} v(\tau)} \int_{T_{2 k}}^{\tau} \frac{-r(s) d s}{r^{2}(s)} \\
& \leqslant 12\left(M^{* 2} / m_{0} v(\tau)\right)(1 / r(\tau))<12 M^{* 2} / m_{0}
\end{aligned}
$$

which contradicts the definition of $\tau$. This proves the lemma.

Notation. Let $V_{i}=C_{i}, V_{i j}=C_{i j}$. Lower case $v$ 's denote absolute value of capital $V$ 's.

Corollary to Lemma 21. We can write for $T_{k-1}<t<T_{k}, V_{p}(t)=$ $V_{p}\left(T_{k-1}\right)+V_{p}^{*}(t)$ where $\left|V_{p}^{*}\right|=v_{p}^{*}<a_{19}$.

Proof. For $t \in\left[T_{k-1}, T_{1 k}\right], v_{p}^{*}(t)<a_{4}\left(T_{1 k}-T_{k-1}\right)$ since $G_{p}$ is one of the clusters on $S_{k-1}$. On $W_{k}$, moreover, $\left|V_{p}(t)-V_{p}\left(T_{1 k}\right)\right|<a_{3} \delta\left(T_{1 k}\right)$. Therefore, 
for $T_{k-1} \leqslant t \leqslant T_{2 k}$,

$$
v_{p}^{*}(t)<a_{4}\left(T_{1 k}-T_{k-1}\right)+a_{3} \delta\left(T_{1 k}\right)<1 .
$$

Finally we use the matrix $A$ of Lemma 14 to write $A\left(V_{s}, V_{p}\right)=\left(V, V_{p^{\prime}}\right)$. If we also borrow the function, $W^{*}(t)=\left(V(t), V_{p^{\prime}}(t)\right)-\left(V\left(T_{2 k}\right), V_{p^{\prime}}\left(T_{2 k}\right)\right)$ for $t \in S_{k}$, Corollary 1 of Lemma 4 and Lemma 21 assert that $\left|W^{*}(t)\right|$ is bounded for $T_{2 k}<t<T_{k}$. It is clear from this that

$$
\begin{aligned}
\left|V_{p}(t)-V_{p}\left(T_{2 k}\right)\right| & <\left|\left(V_{s}(t), V_{p}(t)\right)-\left(V_{s}\left(T_{2 k}\right), V_{p}\left(T_{2 k}\right)\right)\right| \\
& <a_{14}\left|W^{*}(t)\right|<a_{19}^{\prime} .
\end{aligned}
$$

Combining the estimates for the intervals $\left[T_{k-1}, T_{1 k}\right],\left[T_{1 k}, T_{2 k}\right]$, and $\left[T_{2 k}\right.$, $T_{k}$ ], we now have for $T_{k-1} \leqslant t \leqslant T_{k}$,

$$
v_{p}^{*}(t)<a_{4}\left(T_{1 k}-T_{k-1}\right)+a_{3} \delta\left(T_{1 k}\right)+a_{19}^{\prime}<a_{19} \text {. }
$$

8. In the previous section, it was shown that the clusters $H, H^{\prime}$ must be effectively smaller in size than $1 / V\left(T_{k}\right)$. This bound will enable us to show that the sequence of lines $L_{k}$ (see notation below), must converge extremely rapidly. This rapid convergence of the sequence $L_{k}$ to a limiting line, $L$, will force the convergence of the particle positions $R_{i}, i=1,2,3,4$, to the limiting line $L$.

Notation. Let $L_{k}$ be a line defined by the two points $D\left(T_{k}\right), D^{\prime}\left(T_{k}\right)$.

REMARK. $L_{k}$ may be defined equivalently by the pairs of points $D\left(T_{k}\right)$ and 0 ; or $D^{\prime}\left(T_{k}\right)$ and 0 . Therefore, $L_{k-1}$ is also defined by $C_{p}\left(T_{k-1}\right)$ and 0 .

LEMMA 22. We can find a positive number, $a_{22}$, such that $\left|D\left(T_{k}\right)-L_{k-1}\right|<$ $a_{22}\left(T_{k}-T_{k-1}\right)$.

Proof. By Theorem 1, we can restrict our attention to $k$ large enough that $|Z|+\left|Z^{\prime}\right|<1$ for $t \in S_{k-1}$. Now use the cluster formula for angular momentum to express

$$
C=\left(M_{s}+M_{p^{\prime}}\right) D_{-1} \times D_{-1}^{\cdot}+M_{p} D_{-1}^{\prime} \times D_{-1}^{\prime}+Z+Z^{\prime} .
$$

We then rearrange to write

$$
\left|\left(M_{s}+M_{p^{\prime}}\right) D_{-1} \times D_{-1}^{\prime}+M_{p} D_{-1}^{\prime} \times D_{-1}^{\prime}\right|<|C|+1 .
$$

Now let us write $D_{-1}=\left(-M_{p} /\left(M_{s}+M_{p^{\prime}}\right)\right) D_{-1}^{\prime}$ since $M_{p} D_{-1}^{\prime}=M_{p} C_{p}=$ $-M_{s} C_{s}-M_{p^{\prime}} C_{p^{\prime}}=-\left(M_{s}+M_{p^{\prime}}\right) D_{-1}$, and use this to express

$$
\begin{aligned}
\left(M_{s}+M_{p^{\prime}}\right) D_{-1} & \times D_{-1}^{\prime}+M_{p} D_{-1}^{\prime} \times D_{-1}^{\prime} \\
& =\left(M_{p} M^{*} /\left(M_{s}+M_{p^{\prime}}\right)\right) D_{-1}^{\prime} \times D_{-1}^{\prime} .
\end{aligned}
$$

Therefore

$$
\begin{aligned}
\left|D_{-1}^{\prime} \times D_{-1}^{\prime}\right| & =\left|C_{p} \times V_{p}\right|<\left(M_{s}+M_{p^{\prime}}\right)(|C|+1) /\left(M_{p} M^{*}\right) \\
& <(|C|+1) / m_{0}=a_{20} .
\end{aligned}
$$


We now will outline the remainder of the proof of the lemma. We follow $D_{-1}^{\prime}\left(T_{k-1}\right)=C_{p}\left(T_{k-1}\right)$ from $T_{k-1}$ at which time $G_{p}$ comprises part of $H$. We then use the estimate $\left|C_{p}\left(T_{k-1}\right) \times V_{p}\left(T_{k-1}\right)\right| \leqslant a_{20}$ to make $\left|C_{p}\left(T_{k}\right)-L_{k-1}\right|$ small. We use Lemma 21 and the fact that $D\left(T_{k}\right)$ is a convex combination of $C_{p}\left(T_{k}\right), C_{s}\left(T_{k}\right)$ to show that $\left|C_{p}\left(T_{k}\right)-D\left(T_{k}\right)\right|$ is small. We finally use the triangle inequality to show that $\left|D_{k}\left(T_{k}\right)-\left(L_{k-1}\right)\right|$ is small which completes the proof.

Suppose we define a coordinate system in which we express $V_{p}$ in the following way. We choose this coordinate system to have three pairwise orthogonal axes, with the third axis parallel to $L_{k-1}$. Let $u_{1}, u_{2}$ be the first components of $V_{p}$. Then

$$
\left|C_{p}(t)-L_{k-1}\right|<\int_{T_{k-1}}^{t}\left(u_{1}^{2}+u_{2}^{2}\right)^{1 / 2} d t
$$

Let $\rho(t)=\left(u_{1}^{2}+u_{2}^{2}\right)^{1 / 2}$. It is a computation to verify

$$
\rho\left(T_{k-1}\right)=\left|C_{p}\left(T_{k-1}\right) \times V_{p}\left(T_{k-1}\right)\right| / c_{p}\left(T_{k-1}\right) .
$$

We submit

$$
\begin{aligned}
c_{p}\left(T_{k-1}\right) & =\left|D_{-1}^{\prime}\left(T_{k-1}\right)\right| \\
& =\left(1 /\left(1+\left(M_{s}+M_{p^{\prime}}\right) / M_{p}\right)\right)\left|D_{-1}^{\prime}\left(T_{k-1}\right)-D_{-1}\left(T_{k-1}\right)\right| \\
& >m_{0} a_{1} / 3 M^{*}=a_{21} .
\end{aligned}
$$

We then compute $\rho\left(T_{k-1}\right) \leqslant a_{20} / a_{21}$. Let

$$
\rho^{*}(t)=\left[\sum_{1}^{2}\left(u_{i}(t)-u_{i}\left(T_{k-1}\right)\right)^{2}\right]^{1 / 2} .
$$

It is clear that $\rho^{*}(t) \leqslant v_{p}^{*}(t)<a_{19}$ by corollary to Lemma 21 . By the triangle inequality,

$$
\rho(t)<\rho\left(T_{k-1}\right)+\rho^{*}(t) \leqslant a_{19}+a_{20} / a_{21} \text { for } T_{k-1} \leqslant t \leqslant T_{k} .
$$

Therefore

$$
\left|C_{p}\left(T_{k}\right)-L_{k-1}\right| \leqslant \int_{T_{k-1}}^{T_{k}} \rho(s) d s \leqslant\left(a_{19}+\frac{a_{20}}{a_{21}}\right)\left(T_{k}-T_{k-1}\right) .
$$

By the corollary to Lemma 20 and the fact that $D\left(T_{k}\right)$ is a convex combination of $C_{p}\left(T_{k}\right)$ and $C_{s}\left(T_{k}\right)$, we have $\left|C_{p}\left(T_{k}\right)-D\left(T_{k}\right)\right| \leqslant r\left(T_{k}\right) \leqslant$ $1 / v\left(T_{k}\right)$. We have, by Lemma $21, v\left(T_{k}\right)>v\left(T_{2 k}\right)-\left(16 M^{2} / m_{0}\right)$. By the mean value theorem and Lemma 3, 


$$
\begin{aligned}
v\left(T_{k}\right) & >\frac{\left|C_{s p}\left(T_{1 k}\right)-C_{s p}\left(T_{2 k}\right)\right|}{T_{2 k}-T_{1 k}}-2 a_{3} \delta\left(T_{1 k}\right)-\frac{16 M^{* 2}}{m_{0}} \\
& >\frac{a_{1} / 3-\delta\left(T_{2 k}\right)-\left(2 a_{3} \delta\left(T_{1 k}\right)+16 M^{* 2} / m_{0}\right)\left(T_{2 k}-T_{1 k}\right)}{T_{2 k}-T_{1 k}} \\
& >a_{1} / 4\left(T_{2 k}-T_{1 k}\right) .
\end{aligned}
$$

Therefore, $\left|C_{p}\left(T_{k}\right)-D\left(T_{k}\right)\right|<4\left(T_{2 k}-T_{1 k}\right) / a_{1}$. We put the estimate for $\left|C_{p}\left(T_{k}\right)-L_{k-1}\right|$ and the one for $\left|C_{p}\left(T_{k}\right)-D\left(T_{k}\right)\right|$ together with the triangle inequality to give the desired result,

$\left|D\left(T_{k}\right)-L_{k-1}\right|<\left(a_{19}+a_{20} / a_{21}+4 / a_{1}\right)\left(T_{k}-T_{k-1}\right)=a_{22}\left(T_{k}-T_{k-1}\right)$.

Corollary 1. $\left|D^{\prime}\left(T_{k}\right)-L_{k-1}\right|<M^{*} a_{22}\left(T_{k}-T_{k-1}\right) / m_{0}$.

Proof. Since $\left(M_{s}+M_{p}\right) D=-\left(M_{p^{\prime}} D^{\prime}\right)$, then the corollary follows.

COROLlary 2. Let $\theta_{k}$ be the angle between $L_{k}$ and $L_{k-1}$ measured so it will always be positive and less than or equal to $\pi / 2$. Then $\lim _{k \rightarrow \infty} \theta_{k}=0$.

Proof. $\theta_{k}$ is the angle between $D\left(T_{k}\right)$ and $L_{k-1}$, and equals the angle between $D^{\prime}\left(T_{k}\right)$ and $L_{k-1}$. $\theta_{k}$ must tend to 0 by Lemma 22 and $\mid D\left(T_{k}\right)-$ $D^{\prime}\left(T_{k}\right) \mid>a_{1} / 3$.

Notation. Let $\zeta_{k}= \pm\left(D\left(T_{k}\right)-D^{\prime}\left(T_{k}\right)\right)$ and $u_{k}=\zeta_{k} /\left|\zeta_{k}\right|$. We choose the sign of $\zeta_{k}$ and $u_{k}$ such that $u_{k} u_{k+1}$ is positive for large $k$. Corollary 2 of Lemma 22 assures us that this is possible.

LEMMA 23. Let $k^{\prime}>k$; then $\left|\zeta_{k^{\prime}}\right|>m_{0}\left|\zeta_{k}\right| / 4 M^{*}$.

Notation. For clarity, we subscript the sets $H, H^{\prime}$. Let $H_{i}, H_{i}^{\prime}$ be sets, respectively, $H, H^{\prime}$ on $S_{i}$.

Proof. Merely pick $k$ great enough that $t$ larger than $T_{k}$ assures $I \cdot(t)$ positive. We also want

$$
\min _{\substack{i \in H_{k} \\ j \in H_{k}^{\prime}}} r_{i j}\left(T_{k}\right)>\frac{1}{2}\left|\zeta_{k}\right| \text { and } \max _{\substack{i \in H_{k^{\prime}} \\ j \in H_{k^{\prime}}}} r_{i j}\left(T_{k^{\prime}}\right)<2\left|\zeta_{k^{\prime}}\right| \text {. }
$$

This and the formula for $I$ in terms of the mutual distances, $r_{i j}$, implies

$$
I\left(T_{k}\right) \geqslant\left(\frac{1}{4} m_{0}^{2} / M^{*}\right)\left|\zeta_{k}\right|^{2}, \quad I\left(T_{k}^{\prime}\right) \leqslant 4 M^{*}\left|\zeta_{k}\right|^{2} .
$$

Combining the preceding two inequalities with $I\left(T_{k^{\prime}}\right) \geqslant I\left(T_{k}\right)$, we obtain $\left|\zeta_{k^{\prime}}\right| \geqslant \frac{1}{4}\left(m_{0} / M^{*}\right)\left|\zeta_{k}\right|$.

REMARKS. In Lemma 24, we will prove that the centers of mass of the clusters $H$ and $H^{\prime}$ on $S_{k}, D, D^{\prime}$ tend to a line, $L$, at the sequence of times $T_{k}$. To show Theorem 2 from Lemma 24 is not hard. The real work comes in finding the limiting line $L$. We have already done most of this work in Lemmas 20-22. 
LEMMA 24. There exists a line $L$ such that $\lim _{k \rightarrow \infty}\left|D\left(T_{k}\right)-L\right|=$ $\lim _{k \rightarrow \infty}\left|D^{\prime}\left(T_{k}\right)-L\right|=0$.

Proof. It suffices to show $\left|D\left(T_{k}\right)-L\right|$ tends to 0 since

$$
\left|D\left(T_{k}\right)-L\right|=\left(\left|D\left(T_{k}\right)\right| /\left|D^{\prime}\left(T_{k}\right)\right|\right)\left|D^{\prime}\left(T_{k}\right)-L\right| \text {. }
$$

However, $m_{0} / M^{*} \leqslant|D| /\left|D^{\prime}\right| \leqslant M^{*} / m_{0}$. We now try to find $L$. To find $L$ we do the easier problem of finding a limit for the sequence of vectors $u_{k}$. By geometry, $\left|u_{k}-u_{k-1}\right|=2 \sin \left(\theta_{k} / 2\right) \leqslant \theta_{k}$. Also, $\left|D\left(T_{k}\right)-L_{k-1}\right|=$ $\left|D\left(T_{k}\right)\right| \sin \left(\theta_{k}\right)$. By Corollary 2 of Lemma 22, $\theta_{k}$ tends to 0 . We restrict attention to $k$ large enough that $0 \leqslant \theta_{k} \leqslant \pi / 6$. Thus, $\theta_{k} \leqslant 2 \sin \left(\theta_{k}\right)$ by the mean value theorem. Therefore, we can write

$$
\left|u_{k}-u_{k-1}\right| \leqslant\left(2 /\left|D\left(T_{k}\right)\right|\right) a_{22}\left(T_{k}-T_{k-1}\right),
$$

by Lemma 22 . This inequality would show that $\left\{u_{k}\right\}$ is a Cauchy sequence if we know $\left|D\left(T_{k}\right)\right|$ bounded away from 0 since $\left(T_{k}-T_{k-1}\right)$ is summable. We have $\left|\zeta_{k}\right|=\left|D\left(T_{k}\right)\right|+\left|D^{\prime}\left(T_{k}\right)\right|$ since $M_{p^{\prime}} D^{\prime}+\left(M_{s}+M_{p}\right) D=0$. However, $\left|D^{\prime}\right| \leqslant\left(M^{*} / m_{0}\right)|D|$ so $\left|D\left(T_{k}\right)\right| \geqslant\left(1 /\left(1+M^{*} / m_{0}\right)\right)\left|\zeta_{k}\right|$ which is bounded away from 0 since $\left|\zeta_{k}\right|>a_{1} / 3$. Therefore the sequence $\left\{u_{k}\right\}$ converges. Let $u$ be the limit of this sequence and let $L$ be the line $\{\gamma u \mid \gamma \in R\}$. By geometry, we have $\left|D\left(T_{k}\right)-L\right|<\left|\zeta_{k}\right| \cdot\left|u-u_{k}\right|$. Therefore if we can show that $\left|\zeta_{k}\right| \cdot \mid u$ $-u_{k} \mid$ tends to 0 , then we have proved this lemma. We know that

$$
\begin{aligned}
\left|u-u_{k}\right| & \leqslant \sum_{i=k+1}^{\infty}\left|u_{i}-u_{i-1}\right| \leqslant \sum_{i=k+1}^{\infty} \frac{2}{\left|D\left(T_{i}\right)\right|} a_{22}\left(T_{i}-T_{i-1}\right) \\
& \leqslant 2\left(1+\frac{M^{*}}{m_{0}}\right) \sum_{i=k+1}^{\infty} \frac{1}{\left|\zeta_{i}\right|} a_{22}\left(T_{i}-T_{i-1}\right) .
\end{aligned}
$$

By Lemma 23 , we can replace $\left(1 /\left|\zeta_{i}\right|\right)$ by $4\left(M^{*} / m_{0}\right)\left(1 /\left|\zeta_{k}\right|\right)$; so combining all this, we obtain

$$
\begin{aligned}
\left|D\left(T_{k}\right)-L\right| & \leqslant\left|\zeta_{k}\right| \sum_{i=k+1}^{\infty}\left|u_{i}-u_{i-1}\right| \\
& \leqslant \frac{8 M^{*}}{m_{0}}\left(1+\frac{M^{*}}{m_{0}}\right) a_{22} \sum_{i=k+1}^{\infty}\left(T_{i}-T_{i-1}\right)=a_{23}\left(-T_{k}\right)
\end{aligned}
$$

which tends to 0 as was the claim of the lemma.

LEMMA 25. Suppose $f(x)$ is a $C^{1}$ map of some interval $I=\{x \mid \alpha \leqslant x \leqslant \beta\}$ into $R^{n}$. Moreover, suppose $L$ is a line in $R^{n}$ and $|f(\alpha)-L|=b_{1},|f(\beta)-L|$ $=b_{2}$ and $|f \cdot(x)-f \cdot(\alpha)|<b_{3}$ for $b_{1}, b_{2}, b_{3}$ positive numbers. Then

$$
\max _{I}|f(t)-L| \leqslant b_{3}(\beta-\alpha)+\max \left\{b_{1}, b_{2}+b_{3}(\beta-\alpha)\right\} .
$$


Proof. Let $g(x)=f(\alpha)+f \cdot(\alpha)(x-\alpha)$. Then $f(\alpha)=g(\alpha)$ and $|f \cdot-g|<$ $b_{3}$. Therefore $|f-g|<b_{3}(\beta-\alpha)$. Since the points $g(x)$ form a line, we know that

$$
\begin{aligned}
\max |g(x)-L| & <\max \{|g(\alpha)-L|,|g(\beta)-L|\} \\
& <\max \left(b_{1}, b_{2}+b_{3}(\beta-\alpha)\right) .
\end{aligned}
$$

However, since we have established that $|f-g|<b_{3}(\beta-\alpha)$, we can now use the triangle inequality to give

$$
\begin{aligned}
|f(x)-L| & \leqslant \max |g(x)-L|+\max |f(x)-g(x)| \\
& \leqslant b_{3}(\beta-\alpha)+\max \left(b_{1}, b_{2}+b_{3}(\beta-\alpha)\right) .
\end{aligned}
$$

LEMMA 26. $\lim _{t \rightarrow 0}\left|R_{i}(t)-L\right|=0$.

REMARK. This lemma completes the proof of Theorem 2.

Proof. We have shown in Lemma 24 that

$$
\lim _{k \rightarrow \infty}\left|D\left(T_{k}\right)-L\right|=\lim _{k \rightarrow \infty}\left|D^{\prime}\left(T_{k}\right)-L\right|=0 .
$$

For $i \in H, j \in H^{\prime}$, the distances $\left|R_{i}-D\right|$ and $\left|R_{j}-D^{\prime}\right|$ are less than $\max _{i, j \in H, i, j \in H^{\prime}} r_{i j}<2 \delta$ for all $t \in S_{k}$. Therefore we have $\lim _{k \rightarrow \infty}\left|R_{i}\left(T_{k}\right)-L\right|$ $=0, i=1,2,3,4$. We seek to extend this statement to include all $t$ near 0 . We first show that limit of $\left|R_{i}(t)-L\right|$ is 0 as $t$ tends to 0 through $S_{k}$. This will be done by applying Lemma 25 to the function $C_{p}(t)$ on the interval $\left[T_{k-1}, T_{k}\right]$. On $S_{k-1}, G_{p}$ is one of the clusters $H$ or $H^{\prime}$. On $S_{k}, G_{p}$ is contained in $H$. In particular, if $i, j$ both belong to $G_{p}$ then $r_{i j}\left(T_{k-1}\right)<2 \delta\left(T_{k-1}\right)$ and $r_{i j}\left(T_{k}\right)<2 \delta\left(T_{k}\right)$. Therefore

$$
\lim _{k \rightarrow \infty}\left|C_{p}\left(T_{k-1}\right)-L\right|=\lim _{k \rightarrow \infty}\left|C_{p}\left(T_{k}\right)-L\right|=0 .
$$

From the corollary to Lemma 21 for $T_{k-1} \leqslant t \leqslant T_{k}, V_{p}(t)=V_{p}\left(T_{k-1}\right)+V_{p}^{*}$ where $v_{p}^{*}<a_{19}$. Now, by Lemma $25, \lim _{k \rightarrow \infty}\left|C_{p}(t)-L\right|=0$ for all $t \in$ $\left[T_{k-1}, T_{k}\right.$ ]. We will only need this for $T_{k-1} \leqslant t \leqslant T_{1 k}$ and $T_{2 k} \leqslant t \leqslant T_{k}$. Now we observe that for $T_{k-1} \leqslant t \leqslant T_{1 k}$,

$$
\left|D_{-1}(t)-L\right|=\left|C_{p}(t)-L\right|\left|D_{-1}(t)\right| / C_{p}(t) \leqslant\left|C_{p}(t)-L\right|\left(m^{*} / m_{0}\right) .
$$

Therefore the limit of $\left|D_{-1}(t)-L\right|$, as $t$ tends to 0 through the closed intervals $\left[T_{k-1}, T_{1 k}\right.$ ], equals the limit of $\left|C_{p}(t)-L\right|$ as $t$ tends to 0 through the closed intervals $\left[T_{k-1}, T_{1 k}\right]$ which equals 0 . Now since $C_{p}=D_{-1}^{\prime}$ and $D_{-1}$ are the centers of mass of the clusters $H$ and $H^{\prime}$ on $S_{k-1}$, we then have the limit of $\left|R_{i}(t)-L\right|$ as $t$ tends to 0 through the closed intervals $\left[T_{k-1}, T_{1 k}\right.$ ] equals 0 .

We now wish to show the same thing for $t$ approaching 0 through the closed intervals $\left[T_{2 k}, T_{k}\right.$ ]. From above, we have that the limit of $\left|C_{p}(t)-L\right|$, as $t$ tends to 0 through [ $T_{2 k}, T$ ], equals 0 . We also know that for $t \in S_{k}$, 
$\left|C_{p}(t)-D(t)\right| \leqslant C_{s p}(t) \leqslant S(t)$. Therefore we have the limit of $|D(t)-L|$, as $t$ tends to 0 through $\left[T_{2 k}, T_{k}\right.$ ], equals 0 . Similarly,

$$
\left|D^{\prime}-L\right|=|D-L|\left|\left(D^{\prime} /|D|\right)\right|<|D-L|\left(m^{*} / m_{0}\right) \text {. }
$$

Therefore, limit of $|D(t)-L|$, as $t$ tends to 0 through $\left[T_{2 k}, T_{k}\right]$, equals the limit of $\left|D^{\prime}(t)-L\right|$, as $t$ tends to 0 through [ $\left.T_{2 k}, T_{k}\right]$, equals 0 . We therefore have the desired statement, limit of $\left|R_{i}(t)-L\right|$, as $t$ tends to 0 through $S_{k}$, equals 0 . We can finish the proof by showing that the limit of $\left|R_{i}(t)-L\right|$ as $t$ tends to 0 through $W_{k}$ equals 0 . This can be done by using the result for $S_{k}$ which was just proved. That is, we have

$$
\lim _{k \rightarrow \infty}\left|R_{i}\left(T_{1 k}\right)-L\right|=\lim _{k \rightarrow \infty}\left|R_{i}\left(T_{2 k}\right)-L\right|=0 \text {. }
$$

We use Lemma 25 on the functions $C_{i}, i=1,2,3$, in the intervals [ $T_{1 k}, T_{2 k}$ ]. For $t \in W_{k}, \max _{i, j \in G_{q}} r_{i j}(t)<a_{2} S^{2}$ where $q=1,2,3$. Therefore,

$$
\lim _{k \rightarrow \infty}\left|C_{i}\left(T_{1 k}\right)-L\right|=\lim _{k \rightarrow \infty}\left|C_{i}\left(T_{2 k}\right)-L\right|=0 \text {. }
$$

From Lemma 3 the velocities $V_{i}$ satisfy $\left|V_{i}(t)-V_{i}\left(T_{1 k}\right)\right|<a_{3} S\left(T_{1 k}\right)<1$. Therefore we can apply Lemma 25 to obtain limit of $\left|C_{i}(t)-L\right|, i=1,2,3$, as $t$ tends to 0 through $W_{k}$ equals 0 . This immediately gives the desired result since each point $R_{j}$ is less than $a_{2} S^{2}$ from one of the points $C_{i}$ on $W_{k}$. Therefore the limit of $\left|R_{i}(t)-L\right|$ as $t$ approaches 0 through $W_{k}=0$. We have already shown that the limit of $\left|R_{i}(t)-L\right|$ as $t$ approaches 0 through $S_{k}$ equals 0 . Therefore $\lim _{t \rightarrow 0}\left|R_{i}(t)-L\right|=0$.

\section{REFERENCES}

1. P. Painlevé, Lecons sur la théorie analytique des équations differentielles (Stockholm, 1895), Hermann, Paris, 1897.

2. H. von Zeipel, Sur les singularités du problème des $n$ corps, Ark. Mat. Astr. Fys. (32) 4 (1908).

3. H. Sperling, On the real singularities of the n-body problem, J. Reine Angew. Math. 245 (1970). 14-50.

4. J. Mather and R. McGehee, Orbits for the collinear four-body problem which become unbounded in finite time, Battelle Recontres, 1974 (to appear).

5. Donald G. Saari, Singularities of Newtonian gravitational systems, Dynamical Systems, Academic Press, New York, 1973, pp. 479-487.

6. C. L. Siegel and J. K. Moser, Lectures on celestial mechanics, Springer-Verlag, Berlin, 1971, pp. $34-45$.

7. Aurel Wintner, The analytical foundations of celestial mechanics, Princeton Univ. Press, Princeton, N.J., 1941, pp. 251-252.

Department of Mathematics, University of Tennessee, Knoxville, Tennessee 37916 